Research Paper

\title{
Comparative efficacy of 10 Chinese herbal injections combined with GP regimen chemotherapy for patients with advanced NSCLC a systematic review and network meta-analysis
}

\author{
Juan $\mathrm{Li}^{1,2^{*}}$, Guang-Hui Zhu ${ }^{1,2^{*}}$, Tong-Tong Liu ${ }^{1}$, Bo-Wen $\mathrm{Xu}^{1,2}, \mathrm{Jie} \mathrm{Li}^{1}$ \\ 1. Department of Oncology, Guang' anmen Hospital, China Academy of Chinese Medical Sciences. \\ 2. School of Graduates, Beijing University of Chinese Medicine, Beijing. \\ *These authors contributed equally to this work.
}

$\triangle$ Corresponding author: Jie Li, Department of Oncology, Guang'anmen Hospital, China Academy of Chinese Medical Sciences, Beijing, China; E-mail: drjieli2007@126.com; Tel.: 01088001219.

(C) The author(s). This is an open access article distributed under the terms of the Creative Commons Attribution License (https://creativecommons.org/licenses/by/4.0/). See http://ivyspring.com/terms for full terms and conditions.

Received: 2021.08.23; Accepted: 2021.12.05; Published: 2022.01.01

\begin{abstract}
Background: Numerous studies have indicated that some Chinese herbal injections (CHIs) might have a beneficial treatment effect when used in combination with chemotherapy. However, the results of these studies have been inconsistent. The aim of this network meta-analysis (NMA) was to evaluate and compare the clinical efficacy and safety of different $\mathrm{CHIs}$ combined with gemcitabine plus cisplatin (GP) regimen chemotherapy with that of GP regimen chemotherapy alone in the treatment of patients with advanced non-small cell lung cancer (NSCLC).

Materials and Methods: Eight databases were systematically searched to identify randomized clinical trials (RCTs) from the date of inception of the database to August 11, 2021. The primary outcome measures were the objective response rate (ORR) and adverse reactions (including nausea and vomiting, and leukopenia). The secondary outcome measures were median survival time (MST) and quality of life (QOL). The quality of the included studies was assessed using the Cochrane risk of bias tool. Standard pair-wise and Bayesian NMAs were carried out to compare the effectiveness and safety of different $\mathrm{CHls}$ combined with GP regimen chemotherapy using WinBUGS 14 and Stata 15.1 software. Sensitivity analysis and Egger's test were also performed to check robust.

Results: A total of 92 eligible RCTs involving 7,728 patients and $10 \mathrm{CH}$ s were included. The results showed that Kangai injection (KAI), Kanglaite injection (KLT), Aidi injection and Compound Kushen (CKSI) injection displayed obvious advantages in both efficacy and safety. Aidi+GP (79.0\%) showed great advantages of ORR, and $\mathrm{KAI}+\mathrm{GP}$ and $\mathrm{KLT}+\mathrm{GP}$ had the lowest probability in terms of leukopenia (4.4\%) and nausea and vomiting (24.2\%). Besides, KLT+GP was shown to positively affect MST. According to the subgroup analyses, $\mathrm{CHIs}$ might have a limited effect in reducing adverse reactions, and have a similar effect in squamous cell carcinoma and adenocarcinoma.

Conclusions: KAI+GP of adjuvant drugs, Aidi+GP and CKSI+GP of anticancer drugs appeared to be the advantageous treatment options for patients with advanced NSCLC, owing to its superior therapeutic performance and reduced adverse reactions. KLT+GP might prolong survival. Nevertheless, additional results from multicenter trials and high-quality studies will be pivotal in supporting our findings.
\end{abstract}

Key words: Chinese herb injections; NSCLC; GP regimen chemotherapy; network meta-analysis

\section{Introduction}

Lung cancer is a leading cause of malignancyrelated mortality worldwide and has a high morbidity rate that is continuing to rise. Approximately $85 \%$ of lung cancers are non-small cell lung cancer (NSCLC) [1]. In almost $75 \%$ of NSCLC cases, the patient presents with advanced local invasion and metastasis 
during hospital admission diagnosis [2], putting them beyond the stage at which surgical intervention can be applied. Recently, molecular targeted therapy and immunotherapy have emerged as therapeutic options; however, chemotherapy remains the cornerstone of NSCLC treatment [3], especially for patients with advanced (stage III/IV) disease. Gemcitabine combined with cisplatin (GP) regimen is one of the two standard platinum-based chemotherapy drug regimens. Previous research has shown that the overall survival of patients receiving GP regimen chemotherapy is noninferior to that of patients treated with cisplatin/pemetrexed. Moreover, in patients with squamous cell histology, the GP regimen showed a significant improvement in survival compared with cisplatin/pemetrexed ( $\mathrm{n}=473 ; 10.8 \mathrm{vs}$. 9.4 months, respectively) [4]. However, patients who receive GP chemotherapy may experience gastrointestinal reactions, blood toxicity, and other toxic side effects, which affects their quality of life (QOL) and hinders treatment $[5,6]$.

To increase the therapeutic effect and reduce adverse reactions in cancer treatment, drug combinations are often used. Chinese herbal injections (CHIs) are guided by the theoretical system of syndrome differentiation in traditional Chinese medicine (TCM), combined with the purification of modern advanced technology. In the basic theory of TCM, the pathogenesis of lung cancer involves Qi stagnation, blood stasis, phlegm accumulation, and cancer toxins. In China, CHIs have been used as a complementary or alternative therapy to chemotherapy in the treatment of NSCLC [7,8], particularly due to their effects of removing phlegm, blood stasis, and resolving hard lumps. However, to date, no direct head-to-head comparative evidence regarding the optimal $\mathrm{CHI}$ plus GP regimen chemotherapy for NSCLC treatment has been reported; thus, a network meta-analysis (NMA) that aims to compare CHIs is required.

This study classified 11 national standard CHIs according to their indications for the treatment of tumors and lung cancer [9], and 2 CHIs commonly used in NSCLC [10,11]. Finally, 10 CHIs were selected: Aidi injection (Aidi), Compound Kushen injection (CKSI), Kanglaite injection (KLT), Kangai injection (KAI), Brucea javanica Oil Emulsion injection (BJOE), Shenqi Fuzheng injection (SQFZ), Xiaoaiping injection (XAPI), Astragalus polysaccharide injection (API), Lentinan injection (LTNI) and Elemene injection (ELMI). The primary aim of this study was to investigate and rank the treatment efficacy and safety of the aforementioned CHIs by performing an NMA.

\section{Methods}

This study is presented according to the Preferred Reporting Items for Systematic Reviews and Meta-Analyses (PRISMA) guidelines "NMA extended version" [12], and the study is registered with PROSPERO (CRD42020167142). As the materials used in this study had been published previously, ethical approval was not required.

\section{Data sources}

A literature search was performed to identify randomized clinical trials (RCTs) of CHI-assisted treatment of advanced NSCLC. Eight databases were searched including PubMed, the Cochrane Library, EMBASE, Web of Science (ISI), Chinese National Knowledge Infrastructure (CNKI), Chinese Scientific Journals Full-Text Database (VIP), CBM, and Wanfang Data. The literature search was performed by two independent reviewers (Juan $\mathrm{Li}$ and Guang-Hui Zhu). Searches were restricted to original publications from the date of establishment of the database to August 11, 2021. A combination of the following keywords was used: "lung cancer", "lung carcinoma", "non-small cell lung cancer", "NSCLC", "gemcitabine", or "cisplatin", as well as search terms for each of the CHIs. All retrievals were implemented using the Medical Subject Headings (MeSH) and free word. Besides, all related systematic reviews (SRs) and meta-analyses were evaluated, and studies meeting the inclusion criteria were selected from the references. As an example, the electronic strategy for PubMed is shown in Supplementary Figure 1.

\section{Search strategies and selection criteria}

Studies were selected according to the following inclusion criteria: (1) The study was a RCT. (2) The patients were diagnosed with stage III and IV NSCLC according to histopathological and cytological diagnostic criteria, with the tumor-node-metastasis (TNM) classification based on the American Joint Committee on Cancer staging system [13]. (3) The control group received GP regimen chemotherapy alone, while the experimental group was treated with GP regimen chemotherapy combined with $\mathrm{CHIs}$. The $\mathrm{CHI}$ therapeutic interventions included the following 13 intravenous CHIs: Aidi, Toad Venom Injection (TVI), CKSI, Huachansu injection (HCSI), KLT, KAI, BJOE, SQFZ, XAPI, API, LTNI, ELMI and Ginseng Polysaccharide Injection (GPI). (4) Patients had not received any radiotherapy, other chemotherapy, or other Chinese herbs during the study. (5) The study outcome needed to at least include an objective response rate (ORR) or adverse reactions (nausea and vomiting, and leukopenia). 
Duplicates, non-RCTs (including case-control studies and series case reports), unrelated studies (including those on other treatments), abstracts and reviews without specific data, unrelated SRs, and studies with no information regarding the pharmaceutical company or drug number of the $\mathrm{CHI}$ used were all excluded.

\section{Data extraction and quality assessment}

Two researchers (Guang-Hui Zhu and Bo-wen $\mathrm{Xu}$ ) independently extracted the following information from each study: basic information (lead author, publication date, demographic characteristics, and sample size), characteristics of the intervention (type and usage of CHIs, treatment duration, evaluation criteria of clinical efficacy, and supportive treatments such as anti-nausea drugs and granulocyte colony-stimulating factor), and outcomes. The risk of bias of the included studies was assessed by two authors (Juan Li and Tong-Tong Liu) using the RCT bias risk assessment tool recommended by the Cochrane risk-of-bias criteria [14], and cross-checked finally. Any disagreements were resolved by a third reviewer (Jie Li).

\section{Main outcomes}

Tumor response was assessed according to the ORR. According to the World Health Organization (WHO) guidelines for solid tumor responses [15], or the Response Evaluation Criteria in Solid Tumors (RECIST) [16]. Tumors were evaluated as complete response $(\mathrm{CR})$, partial response (PR), stable disease (SD), or progressive disease (PD), with the ORR being equal to CR plus PR. Adverse reactions (adverse drug events or adverse drug reactions) were pooled, including nausea and vomiting, and leukopenia.

\section{Secondary outcomes}

Median survival time (MST) was considered to represent the long-term synergistic efficacy of a combination therapy. Furthermore, the secondary outcomes also included QOL, which was considered to be improved if a patient's Karnofsky Performance status (KPS) score increased by 10 points or more following treatment [17].

\section{Statistical analysis}

The Bayesian NMA was performed using WinBUGS 14 and Stata 15.1 software. Stata software was applied to draw the network evidence map of the NMA, as well as to test the inconsistency (based on the closed loops and node-splitting model). If the difference exhibited statistical significance $(P<0.05)$, the consistency model was used for analysis and the results were sorted. Otherwise, the inconsistency model was used. The odds ratios (ORs) and 95\% confidence intervals (CIs) of the dichotomous variables were used as the effect-quantity indexes. For survival outcomes, MST was presented as a hazard ratio (HR) with 95\% CI. Pairwise meta-analysis was conducted according to heterogeneity. A frequentist framework, random-effects NMA was used to compare all classes of $\mathrm{CHIs}$ for each pre-specified outcome. The surface under the cumulative ranking curves (SUCRA) was used to assess the efficacy of each drug intervention program; interventions with higher SUCRA values were associated with the highest probability of being more effective. The comparison-adjusted funnel plot was completed to evaluate the clinical and methodological heterogeneity. Subgroup analyses were performed following the cycle of chemotherapy, supportive treatment, evaluation criteria, and pathological types of NSCLC to demonstrate the clinical heterogeneity and its influence on the endpoint.

\section{Results}

\section{Study selection and characteristics}

A total of 3,957 relevant studies were identified through systematic searching and previously published meta-analyses. After the removal of duplicates, leaving 1,842 potentially eligible records. A further 1,312 reviews, case reports, and unrelated studies were excluded after the titles and abstracts were read. Next, the full texts of the remaining 530 studies were assessed for eligibility. There were 438 full-text articles that did not meet the inclusion and exclusion criteria, such as Non-RCTs $(n=59)$, Lack of TNM staging $(n=62)$, Lack of manufacturer $(n=82)$, No intravenous drip $(\mathrm{n}=7)$, Mixed other treatment (n $=228$ ) (Figure 1). Finally, 92 studies [18-109] involving 7,728 patients were finally included in this NMA to compare the efficacy and safety of $10 \mathrm{CHIs}$ (Aidi, KLT, CKSI, KAI, BJOE, SQFZ, XAPI, API, LTNI, and ELMI) plus GP regimen chemotherapy. All of the included studies were conducted in China (Table 1).

For each of the CHIs, the number of included studies was as follows: Aidi, 28 trials; KLT, 20 trials; CKSI, 9 trials; KAI, 6 trials; BJOE, 10 trials; SQFZ, 10 trials; XAPI, 4 trials; API, 1 trial; LTNI, 2 trials; ELMI, 1 trial and 1 trial includes Aidi and KAI [27]. A network plot of the main outcomes of the Bayesian analysis is depicted in Figure 2. And the Nodesplitting model resylts for main outcomes showed no difference presented statistically significance $(P>$ $0.05)$, the consistency model was used for analysis (Supplementary Table 2). 


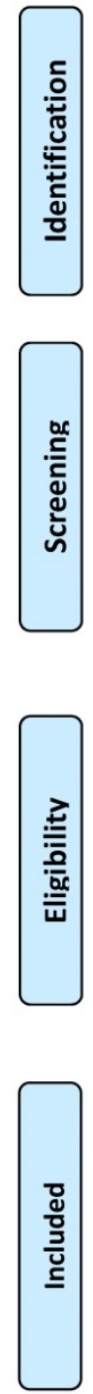

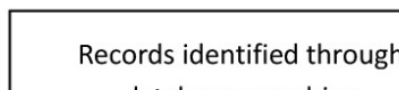

database searching

$(n=3225)$

through previously published

meta-analyses $(n=723)$

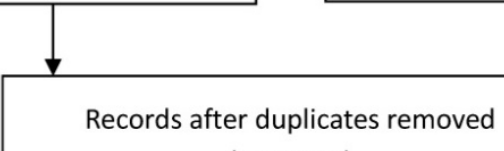

$$
\text { ( } n=2018)
$$
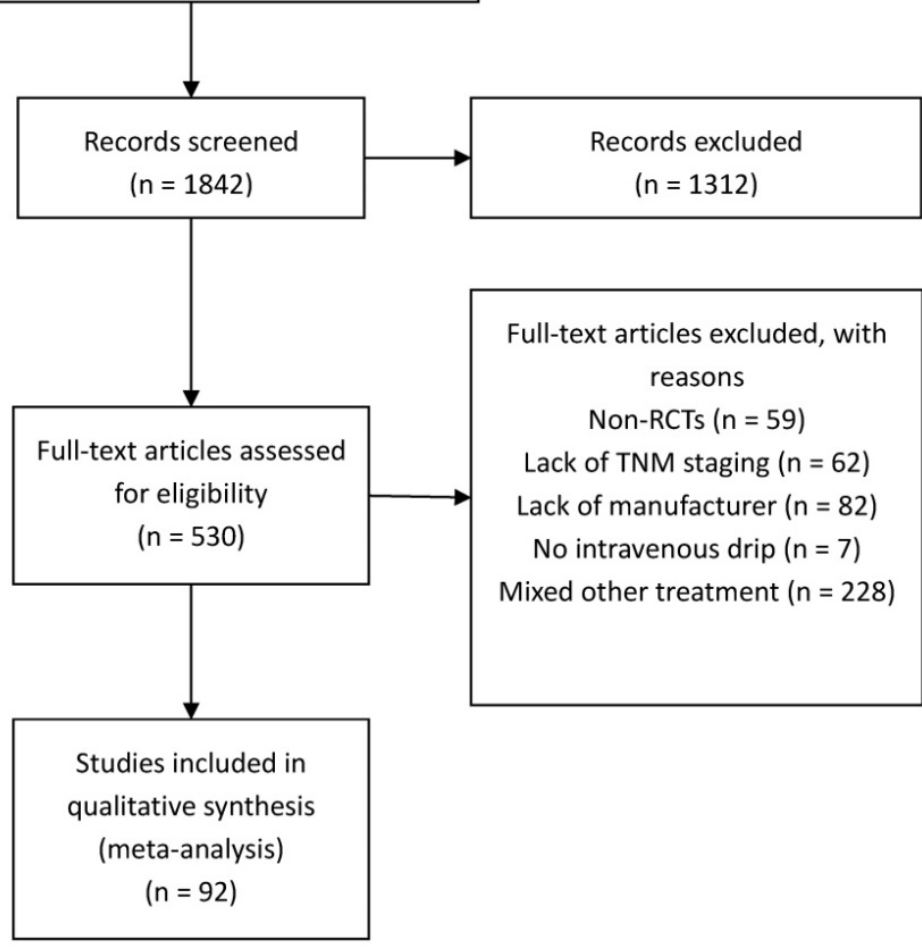

Full-text articles excluded, with reasons

Non-RCTs ( $n=59$ )

Lack of TNM staging ( $n=62$ )

Lack of manufacturer $(n=82)$

No intravenous drip $(n=7)$

Mixed other treatment $(n=228)$

Figure 1. Articles retrieved and assessed for eligibility.

Table 1. Characteristics of included studies

\begin{tabular}{|c|c|c|c|c|c|c|c|c|c|c|c|c|}
\hline \multirow[t]{3}{*}{ First author. year } & \multicolumn{4}{|c|}{ NSCLC (III-IV) } & \multicolumn{3}{|c|}{ Inventions } & \multirow[t]{3}{*}{ Scale (A) } & \multirow[t]{3}{*}{ Scale (B) } & \multirow{3}{*}{$\begin{array}{l}\text { Supportive } \\
\text { treatment }\end{array}$} & \multicolumn{2}{|l|}{ Outcome } \\
\hline & \multirow[t]{2}{*}{$\mathrm{E} / \mathrm{C}$} & \multirow[t]{2}{*}{$\mathrm{M} / \mathrm{F}$} & \multirow[t]{2}{*}{ Age } & \multirow[t]{2}{*}{$\mathrm{Ad} / \mathrm{Sq} / \mathrm{Ot}$} & \multicolumn{2}{|c|}{ Treatment } & \multirow[t]{2}{*}{ Control } & & & & Main & Secondary \\
\hline & & & & & $\mathrm{E}$ & $\mathrm{CHI}(\mathrm{D} / \mathrm{C})$ & & & & & outcomes & outcomes \\
\hline Chen B. 2014 [18] & $49 / 52$ & $78 / 23$ & $27-74$ & $42 / 47 / 12$ & GP+Aidi & $50-100 \mathrm{ml} / 2$ & GP & RECIST & WHO & Yes & (1)(2) (3) & (4) (5) \\
\hline Fu LJ. 2012 [19] & $35 / 35$ & Unclear & $61-84$ & $43 / 26 / 1$ & GP+Aidi & $50 \mathrm{ml} / 2$ & GP & WHO & Unclear & Yes & (1) (2) & \\
\hline Gen KJ. 2020 [20] & $45 / 45$ & $61 / 29$ & $44-79$ & $30 / 50 / 9$ & GP+Aidi & $50 \mathrm{ml} / 4$ & GP & WHO & Unclear & No & (1) & \\
\hline Guo X. 2020 [21] & $51 / 51$ & $58 / 44$ & $43-75$ & Unclear & GP+Aidi & $60 \mathrm{ml} / 4$ & GP & Unclear & Unclear & Yes & (2) (3) & (4) \\
\hline Huang WJ. 2017 [22] & $39 / 40$ & $46 / 33$ & $49-70$ & $27 / 25 / 27$ & GP+Aidi & $60 \mathrm{ml} / 3$ & GP & RECIST & WHO & No & (1) (2) & \\
\hline Kuang XK. 2008 [23] & $26 / 13$ & $33 / 6$ & $34-71$ & $22 / 12 / 5$ & GP+Aidi & $50 \mathrm{ml} / ?$ & GP & WHO & WHO & Yes & (1) (2) & \\
\hline Li J. $2016[24]$ & $47 / 47$ & $52 / 42$ & $40-70$ & $53 / 34 / 7$ & GP+Aidi & $50-100 \mathrm{ml} / 4$ & GP & WHO & WHO & Yes & (1)(2)(3) & \\
\hline Liu H. 2010 [25] & $32 / 32$ & $37 / 27$ & $45-75$ & $35 / 15 / 14$ & GP+Aidi & $50 \mathrm{ml} / 4$ & GP & WHO & Unclear & No & (1)(3) & \\
\hline Liu HF. 2019 [26] & $44 / 44$ & $54 / 34$ & $42-76$ & $39 / 12 / 37$ & GP+Aidi & $50 \mathrm{ml} / 2$ & GP & RECIST & Unclear & No & (1) & \\
\hline Li XY. 2015 [27] & $20 / 20 / 20$ & $24 / 16$ & $45-74$ & $25 / 13 / 2$ & $\begin{array}{l}\text { GP+Aidi } \\
\text { GP+KAI }\end{array}$ & $\begin{array}{l}50 \mathrm{ml} / 2 \\
50 \mathrm{ml} / 2\end{array}$ & GP & Unclear & WHO & No & (2) (3) & (5) \\
\hline Liu YH. 2014 [28] & $43 / 43$ & $53 / 33$ & $39-73$ & $49 / 37 / 0$ & GP+Aidi & $50 \mathrm{ml} / 2$ & GP & $\mathrm{WHO}$ & WHO & Yes & (1)(2) (3) & \\
\hline Мa M. 2017 [29] & $42 / 42$ & $55 / 29$ & $44-75$ & $44 / 9 / 31$ & GP+Aidi & $50 \mathrm{ml} / 4$ & GP & WHO & Unclear & Yes & (1)(2) & \\
\hline Shen RR. 2021 [30] & $30 / 30$ & $40 / 20$ & $34-81$ & $29 / 31 / 0$ & GP+Aidi & $60 \mathrm{ml} / 4$ & GP & WHO & Unclear & No & (1) & \\
\hline Song ZZ. 2009 [31] & $30 / 30$ & $36 / 24$ & $53-76$ & Unclear & GP+Aidi & $50 \mathrm{ml} / 2$ & GP & WHO & WHO & Yes & (1) (3) & (5) \\
\hline Su SJ. 2017 [32] & $40 / 39$ & $45 / 34$ & $40-70$ & $31 / 39 / 9$ & GP+Aidi & $50 \mathrm{ml} / 2$ & GP & RECIST & Unclear & Yes & (1) (2) (3) & \\
\hline Sun GS. 2008 [33] & $33 / 30$ & $54 / 9$ & $34-73$ & $30 / 24 / 9$ & GP+Aidi & $10 \mathrm{ml} / 2$ & GP & WHO & Unclear & Yes & (1) & (4) \\
\hline Sun JB. 2012 [34] & $34 / 34$ & $42 / 26$ & $60-86$ & $41 / 25 / 2$ & GP+Aidi & $50 \mathrm{ml} / 2$ & GP & RECIST & NCI-CTC3 & Yes & (1) (2) (3) & (5) \\
\hline Wang SD. 2015 [35] & $42 / 40$ & $61 / 21$ & $39-67$ & Unclear & GP+Aidi & $50 \mathrm{ml} / 2$ & GP & RECIST & Unclear & No & (1) (2) (3) & \\
\hline Wen HQ. 2014 [36] & $45 / 45$ & $64 / 26$ & $61-81$ & $64 / 23 / 3$ & GP+Aidi & $50 \mathrm{ml} / 2$ & GP & RECIST & NCI-CTC3 & No & (1) (3) & (5) \\
\hline Wen K. 2009 [37] & $38 / 38$ & $52 / 24$ & $32-77$ & $28 / 48 / 0$ & GP+Aidi & $50 \mathrm{ml} / 2$ & GP & WHO & WHO & Yes & (1) (2) (3) & (5) \\
\hline Wu T. 2017 [38] & $67 / 68$ & $83 / 52$ & $43-71$ & $48 / 87$ & GP+Aidi & $100 \mathrm{ml} / 2$ & GP & WHO & Unclear & $\mathrm{NO}$ & (1) (2) & \\
\hline
\end{tabular}




\begin{tabular}{|c|c|c|c|c|c|c|c|c|c|c|c|c|}
\hline \multirow[t]{3}{*}{ First author. year } & \multicolumn{4}{|c|}{ NSCLC (III-IV) } & \multicolumn{3}{|l|}{ Inventions } & \multirow[t]{3}{*}{ Scale (A) } & \multirow[t]{3}{*}{ Scale (B) } & \multirow{3}{*}{$\begin{array}{l}\text { Supportive } \\
\text { treatment }\end{array}$} & Outcome & \\
\hline & E/C & $\mathrm{M} / \mathrm{F}$ & Age & $\mathrm{Ad} / \mathrm{Sq} / \mathrm{Ot}$ & Treatment & & Control & & & & Main & Secondary \\
\hline & & & & & $\mathrm{E}$ & CHI (D/C) & & & & & outcomes & outcomes \\
\hline Xu H. 2013 [39] & $38 / 42$ & $55 / 25$ & $39-81$ & $31 / 49 / 0$ & GP+Aidi & $50 \mathrm{ml} / 2$ & GP & WHO & WHO & No & (1) (2) & (5) \\
\hline Xu Y. 2012 [40] & $33 / 33$ & $36 / 30$ & Unclear & Unclear & GP+Aidi & $80 \mathrm{ml} / 4$ & GP & RECIST & WHO & Yes & (1)(2) (3) & \\
\hline Xu ZJ. 2020 [41] & $40 / 40$ & $53 / 27$ & $49-72$ & $57 / 23 / 0$ & GP+Aidi & $50-100 \mathrm{ml} / 2$ & GP & WHO & Unclear & Yes & (1) (2) & \\
\hline Zhang L. 2009 [42] & $32 / 31$ & $44 / 19$ & $31-79$ & $29 / 27 / 7$ & GP+Aidi & $80 \mathrm{ml} / 2$ & GP & WHO & WHO & Yes & (1) (2) & \\
\hline Zhang XC. 2016 [43] & $25 / 25$ & Unclear & Unclear & Unclear & GP+Aidi & $50 \mathrm{ml} / 4$ & GP & RECIST & WHO & No & (1) & (5) \\
\hline Zhao J. 2019 [44] & $43 / 43$ & $55 / 31$ & Unclear & $39 / 43 / 4$ & GP+Aidi & $50 \mathrm{ml} / 2$ & GP & WHO & Unclear & No & (1) & (4) \\
\hline Zhao S. 2015 [45] & $43 / 43$ & $58 / 28$ & $43-79$ & $36 / 47 / 3$ & GP+Aidi & $100 \mathrm{ml} / 1$ & GP & WHO & WHO & Yes & (1) (2) (3) & (5) \\
\hline Zhou DM. 2018 [46] & $58 / 58$ & $63 / 53$ & $41-70$ & Unclear & GP+Aidi & $50 \mathrm{ml} / 2$ & GP & RECIST & Unclear & No & (1)(2) (3) & \\
\hline Bao H. 2019 [47] & $31 / 31$ & $38 / 24$ & $39-72$ & Unclear & GP+KLT & $200 \mathrm{ml} / 2$ & GP & WHO & Unclear & No & (1) (3) & \\
\hline Chen C. 2018 [48] & $30 / 30$ & Unclear & $35-65$ & Unclear & GP+KLT & $200 \mathrm{ml} / 1$ & GP & RECIST & Unclear & Yes & (1) & \\
\hline Chen W. 2016 [49] & $44 / 44$ & $47 / 41$ & $55-78$ & $58 / 20 / 10$ & GP+KLT & $? / 4$ & GP & RECIST & Unclear & No & (1) & \\
\hline Chen Y. 2018 [50] & $51 / 51$ & $59 / 43$ & $57-79$ & $58 / 28 / 16$ & $\mathrm{GP}+\mathrm{KLT}$ & $200 \mathrm{ml} / 4$ & GP & WHO & Unclear & No & (1) (2) & \\
\hline Guan XQ. 2009 [51] & $12 / 12$ & $11 / 12$ & $36-72$ & $16 / 8 / 0$ & GP+KLT & $300 \mathrm{ml} / 2$ & GP & WHO & NCI-CTC & Yes & (1) (2) (3) & (4) \\
\hline Gui XM. 2020 [52] & $60 / 60$ & $60 / 51$ & $32 / 74$ & $74 / 25 / 21$ & GP+KLT & $200 \mathrm{ml} / 2$ & GP & RECIST & WHO & No & (1) (2) & \\
\hline Huang ZB. 2010 [53] & $35 / 35$ & $44 / 26$ & $59-78$ & $27 / 36 / 7$ & GP+KLT & $200 \mathrm{ml} / 2$ & GP & WHO & WHO & No & (1) (2) & (5) \\
\hline Li HY. 2017 [54] & $41 / 41$ & $43 / 39$ & $55-75$ & $18 / 56 / 8$ & GP+KLT & $? / 4$ & GP & RECIST & Unclear & No & (1) & \\
\hline Liang SG. 2014 [55] & $23 / 20$ & Unclear & $60-75$ & $32 / 16 / 0$ & GP+KLT & $100 \mathrm{ml} / 2$ & GP & WHO & Unclear & No & (1) & (5) \\
\hline Liu F. 2019 [56] & $63 / 63$ & $79 / 47$ & $50-77$ & $67 / 47 / 12$ & GP+KLT & $200 \mathrm{ml} / 2$ & GP & RECIST & Unclear & Yes & (1) & (4) (5) \\
\hline Liu JQ. 2011 [57] & $35 / 35$ & $44 / 26$ & $59-74$ & $27 / 41 / 2$ & GP+KLT & $200 \mathrm{ml} / 2$ & GP & RECIST & WHO & Yes & (1) (2) (3) & (5) \\
\hline Liu Y. 2015 [58] & $43 / 43$ & $55 / 31$ & $42-74$ & $41 / 42 / 3$ & GP+KLT & $200 \mathrm{ml} / 4$ & GP & RECIST & SFDA & Yes & (1) & (5) \\
\hline Long SG. 2017 [59] & $42 / 40$ & $52 / 30$ & $47-70$ & $39 / 22 / 21$ & GP+KLT & $200 \mathrm{ml} / 3$ & GP & RECIST & WHO & No & (1) & \\
\hline Sun SQ. 2012 [60] & $35 / 35$ & $41 / 29$ & $37-75$ & $22 / 38 / 10$ & GP+KLT & $200 \mathrm{ml} / 4$ & GP & WHO & WHO & No & (1) & \\
\hline Wang L. 2014 [61] & $43 / 43$ & $58 / 28$ & $43-79$ & $36 / 47 / 3$ & GP+KLT & $200 \mathrm{ml} / 1$ & GP & RECIST & WHO & Yes & (1) (2) (3) & (5) \\
\hline Wang Y. 2017 [62] & $36 / 36$ & $32 / 40$ & Unclear & $49 / 23 / 0$ & GP+KLT & $60 \mathrm{ml} / 4$ & GP & WHO & Unclear & No & (1) (2) (3) & (5) \\
\hline Yan QH. 2018 [63] & $49 / 49$ & $63 / 35$ & $38-76$ & $51 / 47 / 0$ & GP+KLT & $200 \mathrm{ml} / 4$ & GP & RECIST & Unclear & Yes & (1) (2) & \\
\hline Yao J. 2017 [64] & $70 / 67$ & $78 / 59$ & Unclear & $62 / 66 / 9$ & GP+KLT & $200 \mathrm{ml} / 2$ & GP & RECIST & WHO & No & (1) (2) (3) & \\
\hline Ye CY. 2019 [65] & $40 / 40$ & $54 / 26$ & $55-74$ & $40 / 35 / 5$ & GP+KLT & $200 \mathrm{ml} / 2$ & GP & RECIST & Unclear & No & (1) (3) & \\
\hline Zhang MM. 2019 [66] & $50 / 50$ & $52 / 48$ & Unclear & $0 / 100 / 0$ & GP+KLT & $? / 3$ & GP & RECIST & Unclear & No & (1)(2) (3) & (4) \\
\hline Duan P. 2009 [67] & $72 / 71$ & $88 / 55$ & $36-69$ & $69 / 47 / 27$ & $\mathrm{GP}+\mathrm{CKSI}$ & $20 \mathrm{ml} / 2$ & GP & WHO & WHO & Yes & (1) (2) (3) & \\
\hline Fan QL. 2015 [68] & $63 / 63$ & $74 / 52$ & $27-68$ & $60 / 47 / 19$ & GP+CKSI & $25 \mathrm{ml} / 2$ & GP & WHO & WHO & No & (1) & \\
\hline Fen Q. 2018 [69] & $40 / 40$ & $42 / 38$ & Unclear & $9 / 61 / 10$ & GP+CKSI & $15-20 \mathrm{ml} / 2$ & GP & RECIST & WHO & No & (1) & \\
\hline Gao LJ. 2019 [70] & $30 / 30$ & $32 / 28$ & $25-70$ & $26 / 34 / 0$ & GP+CKSI & $20 \mathrm{ml} / 1$ & GP & RECIST & CTCAE4 & No & (1) (3) & (5) \\
\hline Liu Y. 2009 [71] & $44 / 40$ & $52 / 32$ & $42-76$ & $31 / 53 / 0$ & GP+CKSI & $25 \mathrm{ml} / 2$ & GP & WHO & WHO & Yes & (1)(2) (3) & (5) \\
\hline Lu WL. 2017 [72] & $60 / 600060$ & $68 / 52$ & $50-75$ & $48 / 41 / 31$ & GP+CKSI & $20 \mathrm{ml} / 4$ & GP & WHO & WHO & Yes & (1) (2) & (5) \\
\hline Wang ZX. 2009 [73] & $30 / 30$ & $49 / 11$ & $38-75$ & Unclear & $\mathrm{GP}+\mathrm{CKSI}$ & $30 \mathrm{ml} / 2$ & GP & RECIST & WHO & Yes & (1)(2) (3) & (5) \\
\hline Zhang MY. 2019 [74] & $52 / 48$ & $53 / 47$ & $51-76$ & $60 / 36 / 4$ & GP+CKSI & $20 \mathrm{ml} / 2$ & GP & RECIST & Unclear & No & (1) (2) & (5) \\
\hline Zhou HY. 2011 [75] & $40 / 40$ & $42 / 38$ & $34-76$ & $46 / 32 / 2$ & GP+CKSI & $30 \mathrm{ml} / 3$ & GP & WHO & WHO & No & (1)(2) (3) & (4) \\
\hline Chen HL. 2010 [76] & $45 / 41$ & $62 / 24$ & $38-71$ & $44 / 37 / 5$ & GP+BJOE & $30-40 \mathrm{ml} / 4$ & GP & WHO & WHO & No & (1) (2) (3) & (5) \\
\hline Liu SR. 2019 [77] & $49 / 49$ & $51 / 47$ & $45-88$ & $34 / 34 / 30$ & $\mathrm{GP}+\mathrm{BJOE}$ & $30 \mathrm{ml} / 2$ & GP & WHO & WHO & Yes & (1) & \\
\hline Su BK. 2017 [78] & $29 / 28$ & $42 / 15$ & Unclear & $36 / 20 / 1$ & $\mathrm{GP}+\mathrm{BJOE}$ & $30 \mathrm{ml} / 2$ & GP & Unclear & WHO & Yes & (3) & (5) \\
\hline Tian L. 2017 [79] & $48 / 48$ & $63 / 33$ & $40-81$ & $33 / 50 / 13$ & $\mathrm{GP}+\mathrm{BJOE}$ & $30 \mathrm{ml} / 2$ & GP & WHO & Unclear & Yes & (1) & (5) \\
\hline Wang JH. 2012 [80] & $68 / 68$ & $94 / 42$ & $52-74$ & $37 / 99 / 0$ & $\mathrm{GP}+\mathrm{BJOE}$ & $30 \mathrm{ml} / 4$ & GP & WHO & WHO & Yes & (1) & (5) \\
\hline Wang LC. 2015 [81] & $40 / 40$ & $52 / 28$ & $35-75$ & $34 / 32 / 14$ & $\mathrm{GP}+\mathrm{BJOE}$ & $20-30 \mathrm{ml} / 2$ & GP & WHO & WHO & No & (1) (2) & \\
\hline Wang YZ. 2021 [82] & $39 / 39$ & $52 / 26$ & Unclear & Unclear & $\mathrm{GP}+\mathrm{BJOE}$ & $40 \mathrm{ml} / 2$ & GP & WHO & Unclear & Yes & & \\
\hline Ye HN. 2015 [83] & $54 / 53$ & $68 / 39$ & Unclear & $52 / 34 / 21$ & $\mathrm{GP}+\mathrm{BJOE}$ & $30 \mathrm{ml} / 4$ & GP & RECIST & WHO & Yes & & \\
\hline Yu HW. 2020 [84] & $42 / 42$ & $38-84$ & $39 / 45$ & Unclear & $\mathrm{GP}+\mathrm{BJOE}$ & $30 \mathrm{ml} / 3$ & GP & RECIST & Unclear & No & (1) & \\
\hline Zhang B. 2017 [85] & $39 / 39$ & $54 / 24$ & Unclear & $48 / 23 / 7$ & $\mathrm{GP}+\mathrm{BJOE}$ & $40 \mathrm{ml} / 2$ & GP & WHO & Unclear & Yes & (1) & \\
\hline An AJ. 2014 [86] & $49 / 48$ & $52 / 45$ & $39-76$ & $44 / 41 / 12$ & GP+SQFZI & $250 \mathrm{ml} / 2$ & GP & RECIST & Unclear & No & & (5) \\
\hline He WJ. 2008 [87] & $35 / 35$ & $46 / 24$ & $38-75$ & $32 / 38 / 0$ & GP+SQFZI & $250 \mathrm{ml} / 2$ & GP & WHO & WHO & Yes & (1) (2) & (5) \\
\hline He WX. 2021 [88] & $48 / 48$ & $58 / 38$ & $56-78$ & $44 / 52 / 0$ & GP+SQFZI & $250 \mathrm{ml} / 4$ & GP & WHO & Unclear & No & (1) (2) & \\
\hline Lin CH. 2014 [89] & $32 / 30$ & $40 / 22$ & $41-70$ & $39 / 22 / 3$ & GP+SQFZI & $250 \mathrm{ml} / 2$ & GP & Unclear & WHO & No & & (5) \\
\hline Lou T. 2020 [90] & $40 / 40$ & $58 / 22$ & $>60$ & $44 / 27 / 9$ & GP+SQFZI & $250 \mathrm{ml} / 4$ & GP & WHO & Unclear & No & (1)(2) (3) & \\
\hline Luo BP. 2018 [91] & $48 / 48$ & $61 / 35$ & $33-63$ & $30 / 56 / 10$ & GP+SQFZI & $250 \mathrm{ml} / 2$ & GP & WHO & Unclear & No & (1) (2) & \\
\hline Wang YQ. 2010 [92] & $39 / 37$ & $48 / 28$ & Unclear & Unclear & GP+SQFZI & $250 \mathrm{ml} / 2$ & GP & WHO & WHO & No & & (5) \\
\hline Yao DJ. 2013 [93] & $50 / 50$ & $84 / 16$ & $30-70$ & $73 / 27 / 0$ & GP+SQFZI & $250 \mathrm{ml} / 2$ & GP & WHO & WHO & Yes & (1) (2) & (5) \\
\hline Zhang LM. 2017 [94] & $52 / 52$ & $59 / 45$ & $41-82$ & Unclear & GP+SQFZI & $250 \mathrm{ml} / 2$ & GP & WHO & Unclear & Yes & & \\
\hline Zou T. 2009 [95] & $35 / 35$ & $43 / 27$ & $34-70$ & $42 / 24 / 4$ & GP+SQFZI & $250 \mathrm{ml} / 2$ & GP & WHO & WHO & Yes & (1) (2) (3) & (6) \\
\hline Dong H. 2019 [96] & $56 / 56$ & $72 / 40$ & Unclear & $62 / 42 / 8$ & GP+KAI & $40 \mathrm{ml} / 2$ & GP & WHO & Unclear & No & & \\
\hline Ge CZ. 2011 [97] & $32 / 32$ & $47 / 17$ & $65-80$ & $13 / 49 / 2$ & GP+KAI & $30 \mathrm{ml} / ?$ & GP & WHO & Unclear & Yes & & (6) \\
\hline Jiang H. 2018 [98] & $43 / 42$ & $52 / 33$ & Unclear & $28 / 52 / 5$ & $\mathrm{GP}+\mathrm{KAI}$ & $50 \mathrm{ml} / 2$ & GP & RECIST & CTCAE4 & No & (1) (2) (3) & \\
\hline Lu YZ. 2017 [99] & $73 / 73$ & $83 / 63$ & Unclear & $58 / 72 / 16$ & GP+KAI & $60 \mathrm{ml} / 3$ & GP & WHO & WHO & Yes & (2) (3) & \\
\hline Shang LQ. 2011 [100] & $32 / 31$ & $37 / 26$ & 39-71 & $30 / 29 / 4$ & $\mathrm{GP}+\mathrm{KAI}$ & $50 \mathrm{ml} / 4$ & GP & Unclear & WHO & Yes & & \\
\hline Tao HZ. 2020 [101] & $82 / 82$ & $86 / 78$ & Unclear & Unclear & GP+KAI & $50 \mathrm{ml} / 4$ & GP & Unclear & Unclear & No & & \\
\hline Hu XL. 2017 [102] & $53 / 53$ & $66 / 40$ & $332-74$ & $54 / 39 / 13$ & GP+XAPI & $20 \mathrm{ml} / 4$ & GP & RECIST & WHO & Yes & & (4) \\
\hline Li QL. 2016 [103] & $36 / 36$ & $54 / 18$ & $27-74$ & $28 / 42 / 2$ & GP+XAPI & $40-60 \mathrm{ml} / 2$ & GP & RECIST & WHO & Yes & (1) (2) (3) & (4) (5) \\
\hline Liu JR. 2016 [104] & $30 / 30$ & $31 / 29$ & $40-79$ & $0 / 60 / 0$ & GP+XAPI & $40 \mathrm{ml} / 2$ & GP & RECIST & Unclear & No & & \\
\hline Zhang FY. 2011 [105] & $24 / 24$ & $31 / 17$ & $50-75$ & $30 / 18 / 0$ & GP+XAPI & $40-60 \mathrm{ml} / 2$ & GP & WHO & NCL & No & & (5) \\
\hline Qin ZQ. 2009 [106] & $32 / 32$ & $46 / 18$ & Unclear & $36 / 26 / 2$ & GP+API & $250 \mathrm{mg} / 2$ & GP & WHO & WHO & Yes & (1) (2) (3) & (5) \\
\hline Han L. 2012 [107] & $25 / 26$ & $29 / 22$ & Unclear & $30 / 18 / 3$ & $\mathrm{GP}+\mathrm{LTNI}$ & $2 \mathrm{mg} / 2$ & GP & WHO & WHO & Yes & (1)(2) (3) & \\
\hline Li JJ. 2013 [108] & $30 / 22$ & $31 / 21$ & $43-81$ & $35 / 11 / 6$ & GP+LTNI & $2 \mathrm{mg} / 2$ & GP & WHO & WHO & No & (1) (2) (3) & (5) \\
\hline Chu DJ. 2010 [109] & $41 / 41$ & $48 / 34$ & $32-78$ & $45 / 36 / 1$ & GP+ELMI & $500 \mathrm{mg} / 3$ & GP & RECIST & WHO & Yes & (1)(2) (3) & (4) \\
\hline
\end{tabular}


Note: NSCLC: non-small cell lung cancer; E/C: experimental group /control group; CHI (D/C): dose/cycles; Ad/Sq/Ot: adenocarcinoma/squamous carcinoma/other; GP: cisplatin and gemcitabine; Aidi: Aidi injection; KLT: Kanglaite injection; CKSI: Compound Kushen injection; BJOE: Brucea javanica Oil Emulsion injection; SQFZ: Shenqi Fuzheng injection; KAI: Kangai injection; XAPI: Xiaoaiping injection; API: Astragalus polysaccharide injection; LTNI: Lentinan injection; ELMI: Elemene injection; scale. A: evaluation criteria of tumor response; scale. B: evaluation criteria of adverse reactions; RECIST: response evaluation criteria in solid tumors; NCI-CTC: National Cance Institute Common Toxicity Criteria; (1): ORR = CR+PR; (2): leukopenia; (3): nausea and vomiting; (4): median survival time; (5): Karnofsky performance status (KPS).

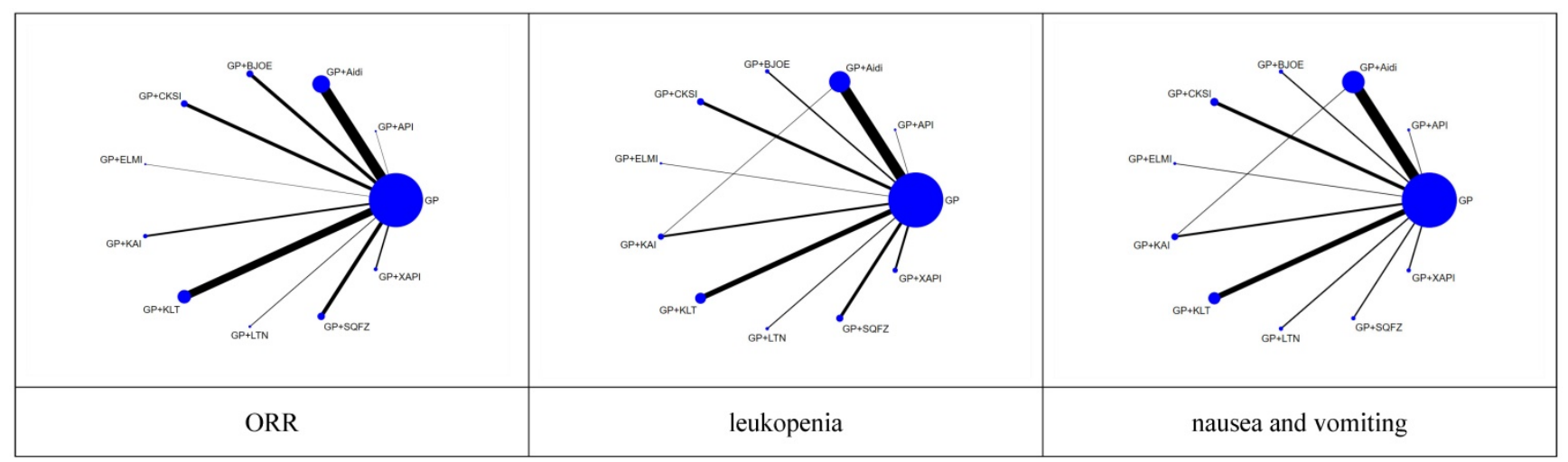

Figure 2. Network graph of the main outcomes. NOTE: Node sizes indicate the total sample sizes for treatments, and the line thickness corresponds to the number of trials.

A

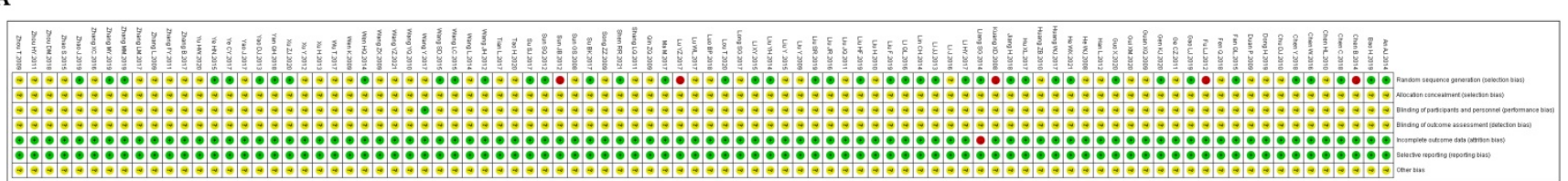

B

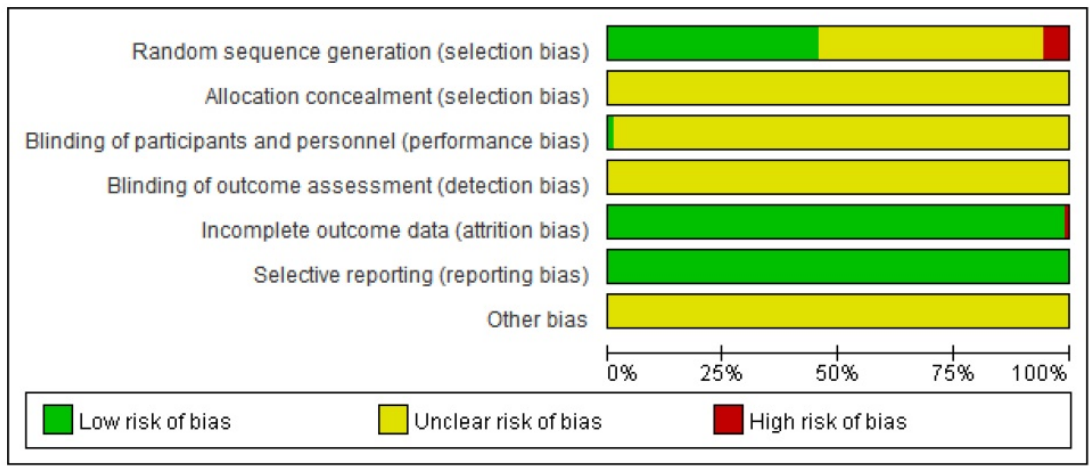

Figure 3. Risk of methodological bias. A. Risk of bias summary: review authors' judgments about each risk of bias item for each included study. B. Risk of bias graph: review authors' judgements about each risk of bias item presented as percentages across all included studies.

There were 41 studies [20-22, 26-30, 32, 35-36, 41, $44,47,49-50,54-56,60,63,65-66,68,70,74,77-78$, $80-81,83,86,88-90,93,98,102,104,108]$ that adopted a random number table, and 2 studies [48, 103] that used direct sampling, to randomize subjects into groups. These studies were rated as having a low risk of bias. Five studies [18-19, 23, 34, 99] were based on the patient's opinion or medical record number, which were rated as having a high risk of bias. None of the included studies reported the details of concealed allocations. One study [62] involved the blinding of patients. Five patients who presented with acute/subacute toxicity withdrew from one study [59]. Besides, in 10 of the studies [33, 49, 51, 54-55, 62, $76,82,85]$, doses were unknown or not recommended. The methodological bias risk of all included studies is presented in Figure 3 and Supplementary Table 1.

\section{Objective response rate (ORR)}

ORR was reported in 88 studies involving 10 CHIs. The results revealed that, compared with the effects of GP regimen chemotherapy alone, GP+Aidi $(\mathrm{OR}=1.87,95 \% \mathrm{CI}[1.56,2.24]), \mathrm{GP}+\mathrm{KLT}(\mathrm{OR}=1.66$, $95 \% \mathrm{CI}[1.35,2.04]), \mathrm{GP}+\mathrm{CKSI}(\mathrm{OR}=1.76,95 \% \mathrm{CI}[1.33$, $2.33]), \mathrm{GP}+\mathrm{BJOE}(\mathrm{OR}=1.70,95 \% \mathrm{CI}[1.28,2.27])$, $\mathrm{GP}+\mathrm{SQFZ}(\mathrm{OR}=1.52,95 \% \mathrm{CI}[1.16,2.01])$, and GP+KAI $(\mathrm{OR}=1.88,95 \% \mathrm{CI}[1.33,2.65])(P<0.05)$ were associated with a significantly improved ORR (Table 2, Supplementary Figure 2). According to the SUCRA analysis of the ORR, the $10 \mathrm{CHIs}$ were ranked as follows: GP+Aidi (79.0\%) > GP+KAI (76.4\%) > $\mathrm{GP}+\mathrm{CKSI}(68.6 \%)>\mathrm{GP}+\mathrm{BJOE}(62.6 \%)>\mathrm{GP}+\mathrm{KLT}$ 
$(60.8 \%)>$ GP+SQFZ $(48.5 \%)>$ GP+ELMI $(44.2 \%)>$ GP+API $(42.5 \%)>$ GP+XAPI $(28.8 \%)>$ GP+LTNI
$(27.1 \%)>$ GP $(11.5 \%)$ (Figure 4$)$.

ORR
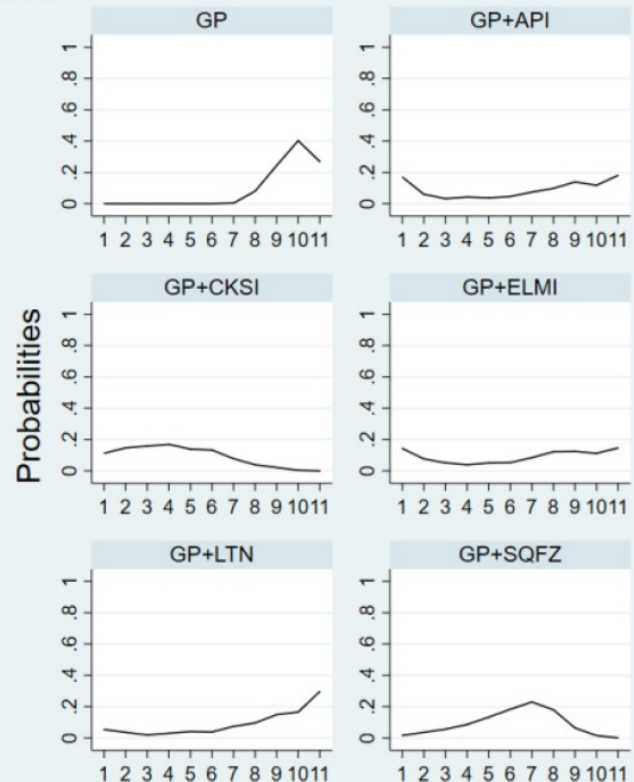

Graphs by Treatment
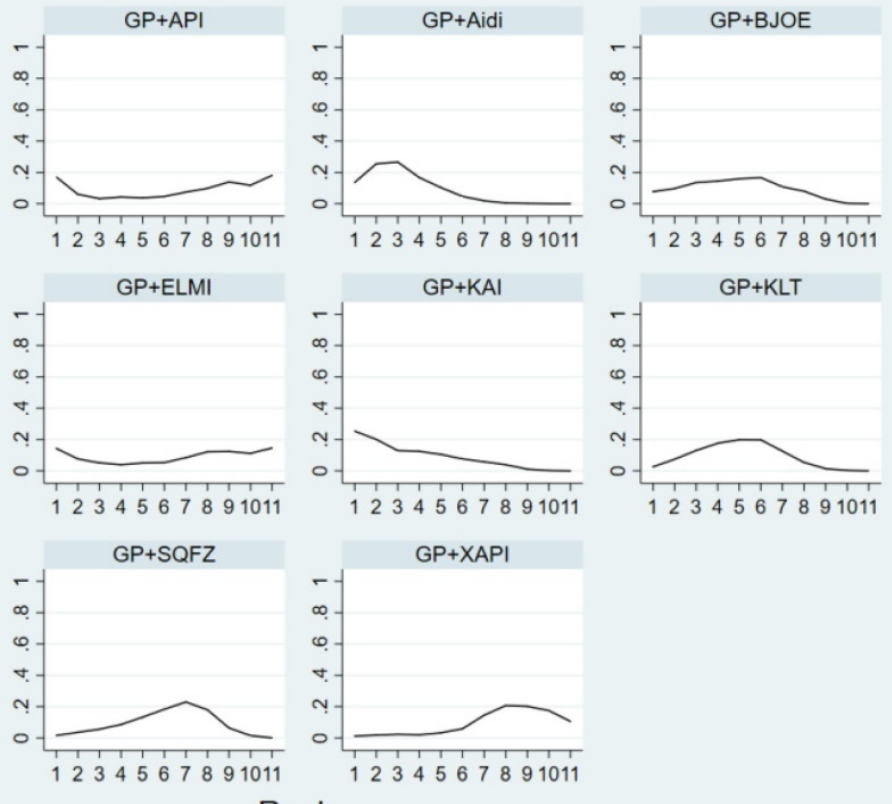

Rank

\section{KPS}
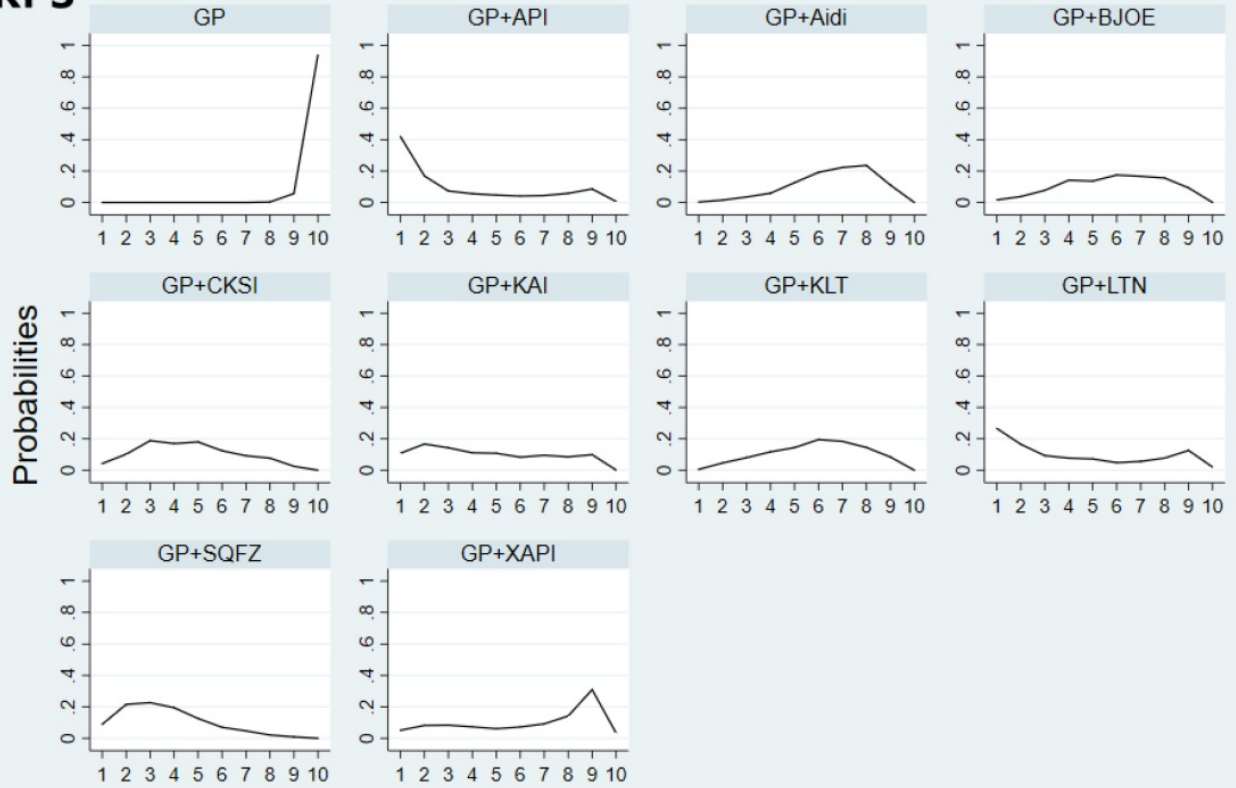

Graphs by Treatment

Rank

Figure 4. Rank of the cumulative probabilities for the ORR and KPS. NOTE: Higher surface under the cumulative ranking curves (SUCRA) values indicated higher probabilities that the treatments were more effective and superior than other therapies.

\section{Adverse reactions}

Fifty-six studies involving $10 \mathrm{CHIs}$ reported on leukopenia. The results showed that GP+Aidi $(\mathrm{OR}=$ $0.40,95 \% \mathrm{CI}[0.30,0.52])$, GP+KLT (OR $=0.44,95 \% \mathrm{CI}$ $[0.31,0.64]), \mathrm{GP}+\mathrm{CKSI}(\mathrm{OR}=0.37,95 \% \mathrm{CI}[0.34,0.55])$,
$\mathrm{GP}+\mathrm{BJOE}(\mathrm{OR}=0.55,95 \% \mathrm{CI}[0.31,0.97]), \mathrm{GP}+\mathrm{SQFZ}$ $(\mathrm{OR}=0.45,95 \% \mathrm{CI}[0.31,0.67]), \mathrm{GP}+\mathrm{KAI}(\mathrm{OR}=0.13$, $95 \% \mathrm{CI}[0.07,0.23]), \mathrm{GP}+\mathrm{XAPI}(\mathrm{OR}=0.48,95 \% \mathrm{CI}[0.29$, $0.79]), \mathrm{GP}+\mathrm{API}(\mathrm{OR}=0.20,95 \% \mathrm{CI}[0.07,0.59])$, GP+LTNI $(\mathrm{OR}=0.29,95 \% \mathrm{CI}[0.11,0.75])$ and GP+ELMI $(\mathrm{OR}=0.23,95 \% \mathrm{CI}[0.09,0.59])(P<0.05)$ 
were associated with lower rates of leukopenia than GP regimen chemotherapy alone. Besides, GP+KAI carried a lower risk of leukopenia than GP+SQFZ (OR $=0.28,95 \% \mathrm{CI}[0.14,0.56]), \mathrm{GP}+\mathrm{BJOE}(\mathrm{OR}=0.23$, $95 \% \mathrm{CI}[0.10,0.52]), \mathrm{GP}+\mathrm{CKSI}(\mathrm{OR}=0.34,95 \% \mathrm{CI}[0.17$, $0.69]), \mathrm{GP}+\mathrm{KLT}(\mathrm{OR}=0.28,95 \% \mathrm{CI}[0.14,0.56])$, and GP+Aidi $(\mathrm{OR}=0.31,95 \% \mathrm{CI}[0.17,0.60])(P<0.05)$. $\mathrm{GP}+\mathrm{XAPI}$ was observed to have a higher risk of leukopenia than GP+KAI (OR $=3.82$, 95\%CI [1.76, 8.28]) $(P<0.05)$ (Table 3, Supplementary Figure 3). Based on the SUCRA analysis of different schemes intervene leukopenia, GP+KAI may be a lower risk treatment option for endpoint events. In terms of the risk of endpoint events, the treatment options were ranked as follows: GP+KAI $(4.4 \%)<\mathrm{GP}+\mathrm{API}(20.0 \%)$ $<$ GP+ELMI $(24.0 \%)<$ GP+LTNI $(36.0 \%)<$ GP+CKSI $(46.3 \%)<$ GP+Aidi (52.2\%) < GP+KLT $(61.9 \%)<$ GP+SQFZ $(63.2 \%)<$ GP+XAPI $(67.0 \%)<$ GP+BJOE $(75.3 \%)<$ GP $(99.8 \%)$ (Figure 5).

Forty studies involving $10 \mathrm{CHIs}$ reported that the GP regimen involved a higher risk of nausea and vomiting than GP+Aidi ( $\mathrm{OR}=2.09,95 \% \mathrm{CI}$ [1.54, $2.83])$, GP+KLT $(\mathrm{OR}=2.82,95 \% \mathrm{CI}[1.74,4.48])$,
$\mathrm{GP}+\mathrm{CKSI}(\mathrm{OR}=1.87,95 \% \mathrm{CI}[1.13,3.09])$ and $\mathrm{GP}+\mathrm{KAI}$ $(\mathrm{OR}=2.50,95 \% \mathrm{CI}[1.29,4.84])(P<0.05)$. Furthermore, $\mathrm{GP}+\mathrm{BJOE}$ carried a higher risk than GP+KLT (OR = 0.37, 95\% CI [0.15, 0.99]) $(P<0.05)$ (Table 3, Supplementary Figure 4). In terms of the risk of nausea and vomiting as an adverse effect, the CHIs were ranked as follows: GP+KLT $(24.2 \%)<\mathrm{GP}+\mathrm{API}$ $(31.3 \%)<$ GP+KAI $(35.8 \%)<$ GP+ELMI $(39.0 \%)<$ GP+XAPI $(41.0 \%)<$ GP+LTNI $(45.5 \%)<$ GP+Aidi $(48.3 \%)<$ GP+SQFZ $(49.5 \%)<$ GP+CKSI $(56.9 \%)<$ GP+BJOE $(85.4 \%)<$ GP $(93.1 \%)$ (Figure 5).

\section{Effects on secondary outcomes}

For secondary outcomes, 11 studies [18, 21, 32, 42, 49, 54, 64, 73, 96-97, 104] reported the MST, involving Aidi, KLT, XAPI, CKSI and ELMI (Supplementary Figure 5). The NMA demonstrated that, when compared with the GP regimen alone, GP+KLT $(\mathrm{HR}=1.45,95 \% \mathrm{CI}[1.12,1.86])(P<0.05)$ was the only combination that showed a statistical difference. Based on the probabilistic collation table, GP+KLT performed best with regard to MST (Supplementary Figure 6).

Table 2. Results of the network meta-analysis of KPS (upper right quadrant) and ORR (lower left quadrant)

\begin{tabular}{|c|c|c|c|c|c|c|c|c|c|c|}
\hline GP+ELMI & GP+LTNI & $\mathrm{GP}+\mathrm{API}$ & GP+XAPI & $\mathrm{GP}+\mathrm{KAI}$ & GP+SQFZ & GP+BJOE & $\mathrm{GP}+\mathrm{CKSI}$ & GP+KLT & GP+Aidi & GP \\
\hline GP+ELMI & NA & NA & NA & NA & NA & NA & NA & NA & NA & NA \\
\hline $1.25(0.38,4.07)$ & GP+LTNI & $1.20(0.23,6.42)$ & $0.68(0.15,2.97)$ & $0.88(0.21,3.64)$ & $0.99(0.28,3.46)$ & $0.76(0.22,2.69)$ & $0.88(0.25,3.10)$ & $0.76(0.22,2.62)$ & $0.71(0.21,2.45)$ & $0.29(0.09,0.94)$ \\
\hline $1.03(0.27,3.92)$ & $0.82(0.23,3.00)$ & $\mathrm{GP}+\mathrm{API}$ & $0.56(0.13,2.46)$ & $0.73(0.18,3.02)$ & $0.82(0.24,2.87)$ & $0.63(0.18,2.23)$ & $0.73(0.21,2.57)$ & $0.63(0.18,2.18)$ & $0.59(0.17,2.03)$ & $0.24(0.07,0.78)$ \\
\hline $1.12(0.41,3.04)$ & $0.90(0.35,2.28)$ & $1.09(0.35,3.35)$ & GP+XAPI & $1.30(0.40,4.23)$ & $1.46(0.55,3.86)$ & $1.13(0.42,3.01)$ & $1.30(0.49,3.46)$ & $1.12(0.43,2.92)$ & $1.05(0.41,2.71)$ & $0.42(0.17,1.02)$ \\
\hline $0.72(0.28,1.83)$ & $0.57(0.24,1.37)$ & $0.70(0.24,2.03)$ & $0.64(0.35,1.16)$ & GP+KAI & $1.12(0.47,2.71)$ & $0.87(0.36,2.11)$ & $1.00(0.41,2.43)$ & $0.86(0.36,2.05)$ & $0.81(0.36,1.85)$ & $0.33(0.15,0.71)$ \\
\hline $0.88(0.35,2.20)$ & $0.71(0.30,1.64)$ & $0.86(0.30,2.45)$ & $0.79(0.45,1.38)$ & $1.23(0.79,1.92)$ & $\mathrm{GP}+\mathrm{SQFZ}$ & $0.77(0.43,1.39)$ & $0.89(0.50,1.60)$ & $0.76(0.44,1.33)$ & $0.72(0.43,1.22)$ & $0.29(0.19,0.44)$ \\
\hline $0.79(0.32,1.98)$ & $0.63(0.27,1.47)$ & $0.77(0.27,2.20)$ & $0.71(0.40,1.24)$ & $1.10(0.70,1.73)$ & $0.89(0.60,1.33)$ & GP+BJOE & $1.16(0.64,2.10)$ & $0.99(0.56,1.76)$ & $0.94(0.54,1.61)$ & $0.38(0.24,0.58)$ \\
\hline $0.76(0.31,1.91)$ & $0.61(0.26,1.42)$ & $0.74(0.26,2.13)$ & $0.68(0.39,1.19)$ & $1.07(0.68,1.66)$ & $0.87(0.58,1.28)$ & $0.97(0.65,1.44)$ & $\mathrm{GP}+\mathrm{CKSI}$ & $0.86(0.49,1.51)$ & $0.81(0.47,1.38)$ & $0.32(0.21,0.49)$ \\
\hline $0.81(0.33,1.98)$ & $0.65(0.28,1.48)$ & $0.79(0.28,2.21)$ & $0.72(0.43,1.22)$ & $1.13(0.76,1.69)$ & $0.92(0.65,1.29)$ & $1.03(0.72,1.46)$ & $1.06(0.75,1.50)$ & GP+KLT & $0.95(0.57,1.57)$ & $0.38(0.26,0.56)$ \\
\hline $0.72(0.29,1.75)$ & $0.57(0.25,1.30)$ & $0.70(0.25,1.96)$ & $0.64(0.38,1.08)$ & $1.00(0.68,1.48)$ & $0.81(0.59,1.13)$ & $0.91(0.65,1.27)$ & $0.94(0.67,1.31)$ & $0.89(0.68,1.16)$ & GP+Aidi & $0.40(0.29,0.56)$ \\
\hline $1.34(0.56,3.22)$ & $1.08(0.49,2.39)$ & $1.31(0.47,3.60)$ & $1.20(0.74,1.95)$ & $1.88(1.33,2.65)$ & $1.52(1.16,2.01)$ & $1.70(1.28,2.27)$ & $1.76(1.33,2.33)$ & $1.66(1.35,2.04)$ & $1.87(1.56,2.24)$ & GP \\
\hline
\end{tabular}

Table 3. Results of the network meta-analysis of nausea and vomiting (upper right quadrant) and leukopenia (lower left quadrant)

\begin{tabular}{|c|c|c|c|c|c|c|c|c|c|c|}
\hline GP+ELMI & GP+LTNI & GP+API & GP+XAPI & GP+KAI & $\mathrm{GP}+\mathrm{SQFZ}$ & GP+BJOE & $\mathrm{GP}+\mathrm{CKSI}$ & GP+KLT & GP+Aidi & GP \\
\hline GP+ELMI & $1.12(0.25,5.02)$ & $0.87(0.16,4.56)$ & $1.07(0.24,4.77)$ & $0.97(0.24,3.84)$ & $1.18(0.28,5.01)$ & $2.31(0.53,10.20)$ & $1.29(0.35,4.80)$ & $0.86(0.23,3.16)$ & $1.16(0.33,4.03)$ & $2.41(0.72,8.11)$ \\
\hline $0.79(0.21,3.00)$ & GP+LTNI & $0.77(0.18,3.27)$ & $0.96(0.27,3.32)$ & $0.86(0.29,2.61)$ & $1.06(0.32,3.45)$ & $2.07(0.60,7.09)$ & $1.16(0.42,3.20)$ & $0.77(0.28,2.10)$ & $1.03(0.41,2.64)$ & $2.16(0.89,5.23)$ \\
\hline $1.14(0.27,4.79)$ & $1.45(0.35,6.08)$ & $\mathrm{GP}+\mathrm{API}$ & $1.24(0.29,5.20)$ & $1.12(0.30,4.17)$ & $1.37(0.34,5.45)$ & $2.68(0.64,11.12)$ & $1.49(0.43,5.19)$ & $0.99(0.29,3.41)$ & $0.41,4.34)$ & $.89,8.71)$ \\
\hline $0.48(0.16,1.39)$ & $0.61(0.21,1.76)$ & $0.42(0.13,1.36)$ & GP+XAPI & $0.90(0.30,2.70)$ & & $2.17(0.64,7.37)$ & & & & $.94,5.42)$ \\
\hline $1.82(0.60,5.56)$ & $2.31(0.76,7.06)$ & $1.59(0.47,5.43)$ & $3.82(1.76,8.28)$ & GP+KAI & $1.23(0.44,3.42)$ & $2.40(0.81,7.05)$ & $1.34(0.58,3.07)$ & $0.89(0.39,2.01)$ & $1.20(0.58,2.46)$ & $2.50(1.29,4.84)$ \\
\hline $0.51(0.18,1.41)$ & $0.64(0.23,1.79)$ & $0.44(0.14,1.39)$ & $1.06(0.56,2.00)$ & $0.28(0.14,0.56)$ & $\mathrm{GP}+\mathrm{SQFZ}$ & $.61,6.23)$ & $1.09(0.43,2.77)$ & $0.29,1.81)$ & $0.98(0.42,2.26)$ & $2.04(0.93,4.46)$ \\
\hline $0.42(0.14,1.25)$ & $0.53(0.18,1.59)$ & $0.36(0.11,1.23)$ & $0.87(0.41,1.85)$ & $0.23(0.10,0.52)$ & $0.82(0.41,1.63)$ & GP+BJOE & $0.56(0.21,1.51)$ & $0.37(0.14,0.99)$ & $0.50(0.20$ & $1.04(0.44,2.45)$ \\
\hline $0.62(0.22,1.72)$ & $0.78(0.28,2.18)$ & $0.54(0.17,1.70)$ & $1.30(0.69,2.44)$ & $0.34(0.17,0.69)$ & $1.22(0.70,2.12)$ & $1.49(0.75,2.96)$ & GP+CKSI & $0.66(0.33,1.33)$ & $0.89(0.50,1.61)$ & $1.87(1.13,3.09)$ \\
\hline $0.51(0.19,1.40)$ & $0.65(0.24,1.78)$ & $0.45(0.14,1.39)$ & $1.07(0.58,1.98)$ & $0.28(0.14,0.56)$ & $1.01(0.59,1.71)$ & $1.23(0.63,2.40)$ & $0.83(0.49,1.40)$ & $\mathrm{GP}+\mathrm{KLT}$ & $1.35(0.76,2.39)$ & $2.82(1.74,4.58)$ \\
\hline $0.57(0.21,1.53)$ & $0.73(0.27,1.94)$ & $0.50(0.17,1.51)$ & $1.20(0.68,2.10)$ & $0.31(0.17,0.60)$ & $1.13(0.71,1.80)$ & $1.38(0.74,2.56)$ & $0.93(0.58,1.47)$ & $1.12(0.72,1.74)$ & GP+Aidi & $2.09(1.54,2.83)$ \\
\hline $\begin{array}{l}0.23 \\
(0.09,0.59)\end{array}$ & $\begin{array}{l}0.29 \\
(0.11,0.75)\end{array}$ & $\begin{array}{l}0.20 \\
(0.07,0.59)\end{array}$ & $0.48(0.29,0.79)$ & $0.13(0.07,0.23)$ & $0.45(0.31,0.67)$ & $0.55(0.31,0.97)$ & $0.37(0.25,0.55)$ & $0.45(0.31,0.64)$ & $0.40(0.31,0.52)$ & GP \\
\hline
\end{tabular}

Note: GP: cisplatin and gemcitabine; Aidi: Aidi injection; KLT: Kanglaite injection; CKSI: Compound Kushen injection; BJOE: Brucea javanica Oil Emulsion injection; SQFZ: Shenqi Fuzheng injection; KAI: Kangai injection; XAPI: Xiaoaiping injection; API: Astragalus polysaccharide injection. 


\section{Leukopenia}
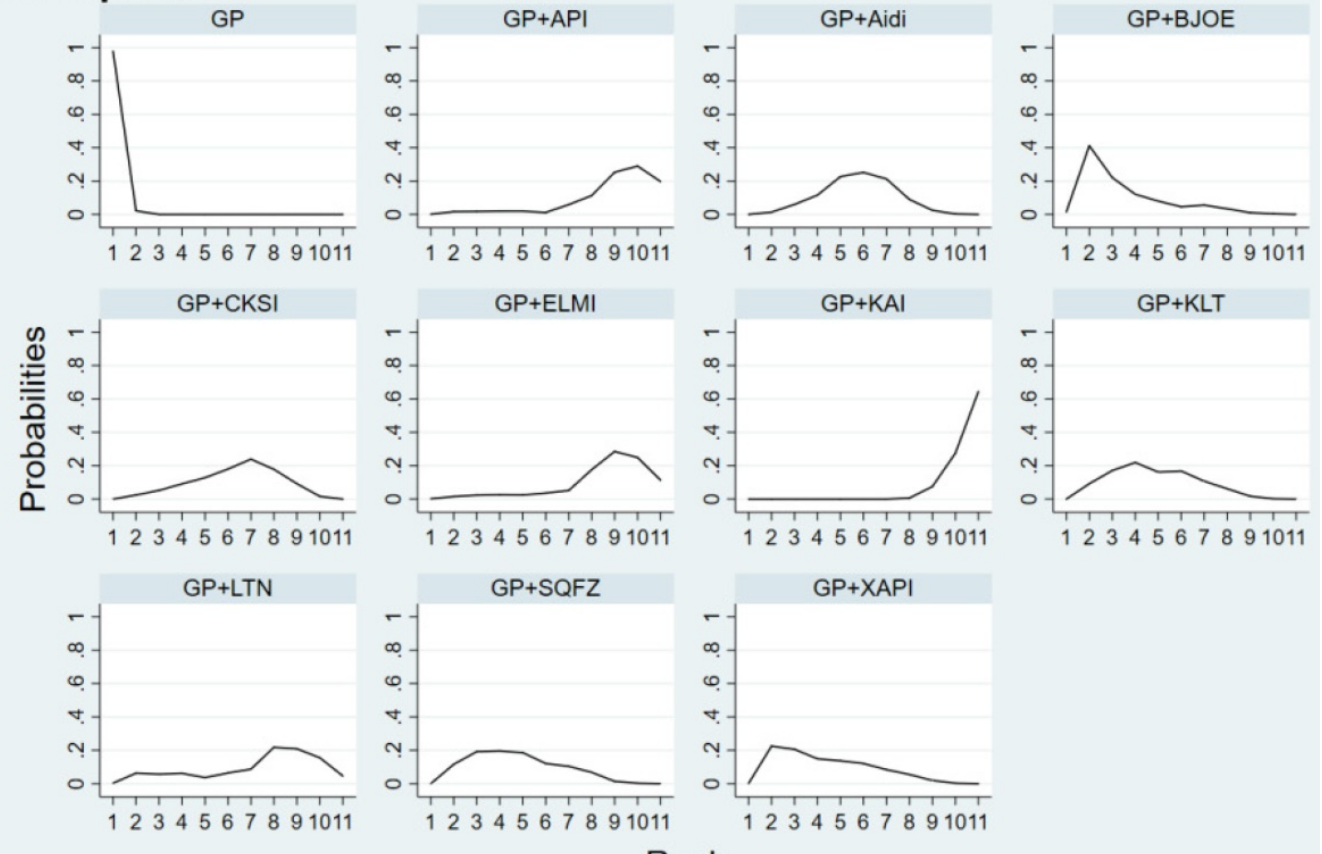

Graphs by Treatment

Rank

\section{Nausea and Vomiting}
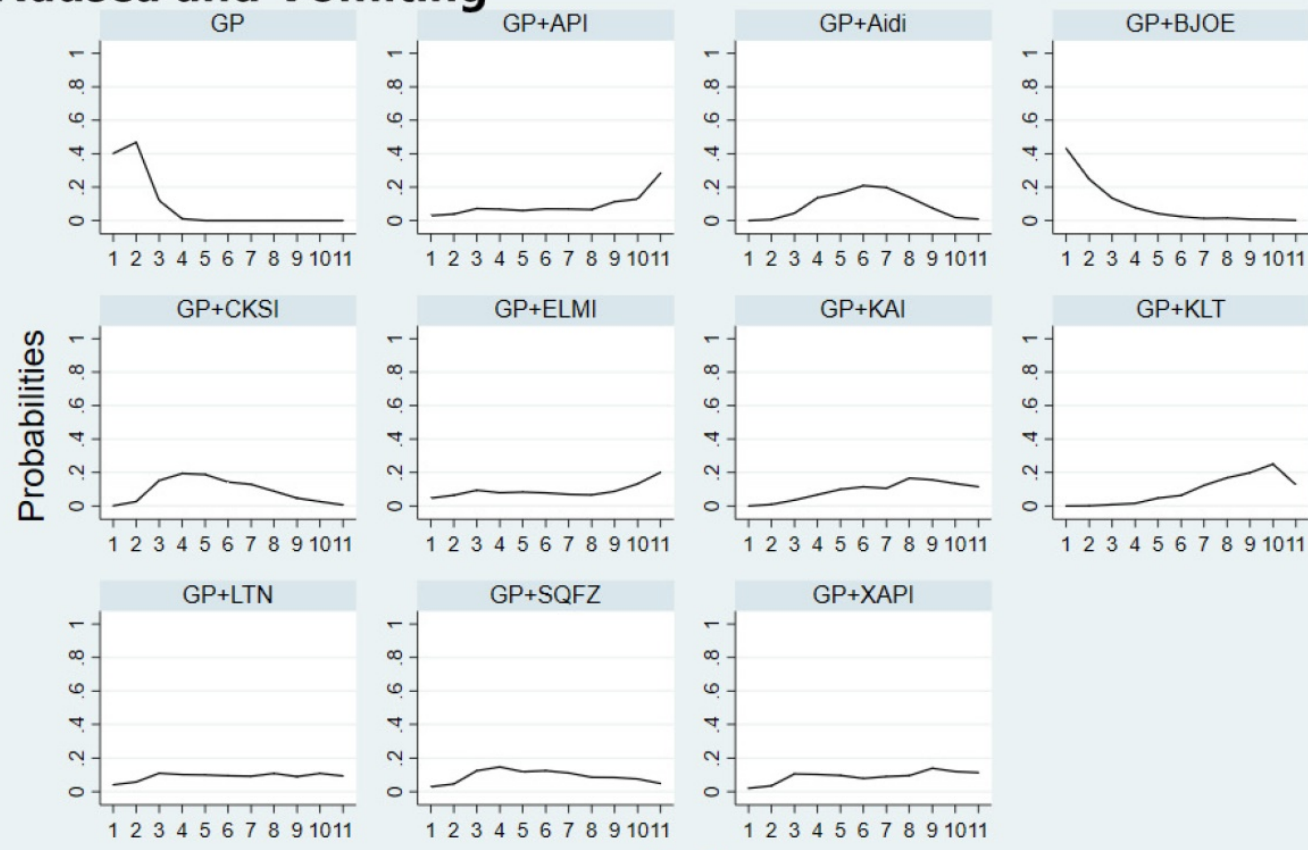

Graphs by Treatment

Rank

Figure 5. Rank of the cumulative probabilities for the leukopenia, nausea and vomiting. NOTE: Higher surface under the cumulative ranking curves (SUCRA) values indicated higher probabilities that the treatments had more adverse reactions than other therapies.

KPS score was reported in 37 studies involving 9 CHIs (Supplementary Figure 5). The results revealed that GP regimen chemotherapy was associated with lower KPS scores than GP+Aidi (OR $=0.40,95 \% \mathrm{CI}$ $[0.29,0.56]), \mathrm{GP}+\mathrm{KLT}(\mathrm{OR}=0.38,95 \% \mathrm{CI}[0.26,0.56])$, $\mathrm{GP}+\mathrm{CKSI}(\mathrm{OR}=0.32,95 \% \mathrm{CI}[0.21,0.49]), \mathrm{GP}+\mathrm{BJOE}$
$(\mathrm{OR}=0.38,95 \% \mathrm{CI}[0.24,0.58]), \mathrm{GP}+\mathrm{SQFZ}(\mathrm{OR}=0.29$, $95 \% \mathrm{CI}[0.19,0.44]), \mathrm{GP}+\mathrm{KAI}(\mathrm{OR}=0.33,95 \% \mathrm{CI}[0.15$, $0.71]), \mathrm{GP}+\mathrm{API}(\mathrm{OR}=0.24,95 \% \mathrm{CI}[0.07,0.78])$ and GP+LTNI $(\mathrm{OR}=0.29,95 \% \mathrm{CI}[0.09,0.94])(P<0.05)$ (Table 2). According to the SUCRA of KPS, the 9 CHIs were ranked as follows: GP+API (74.6\%) > GP+SQFZ 
$(71.0 \%)>$ GP+LTNI $(64.7 \%)>$ GP+KAI $(60.0 \%)>$ GP+CKSI $(59.8 \%)>$ GP+BJOE $(45.8 \%)>\mathrm{GP}+\mathrm{KLT}$ $(45.6 \%)>$ GP+XAPI $(40.0 \%)>$ GP+Aidi $(37.7 \%)>$ GP $(0.7 \%)$ (Figure 4$)$. The SUCRAs of the different treatments outcomes are shown in Table 4.

\section{Cluster analysis}

We used cluster analysis to comprehensively compare the effects of the interventions on two different outcomes. The first cluster analysis was performed for 9 types of CHIs for which ORR and KPS were reported. The plot was based on the SUCRA values of the $\mathrm{CHI}$ groups; each color represents a set of treatment groups that belong to the same cluster, and treatment groups that were located in the upper right corner were superior to other CHIs in both the ORR and KPS. The results of the cluster analysis demonstrated that GP+Aidi, GP+KAI and GP+CKSI had better therapeutic effects than others. Besides, three cluster analyses were conducted for 10 types of CHIs. GP+KAI had advantages in the ranking of ORR and leukopenia and was found to be a tolerable option for the relief of ADRs. As for ORR and nausea, KLT+GP showed better advantages in ranking.

Table 4. Surface under the cumulative ranking curve results of the outcomes

\begin{tabular}{|c|c|c|c|c|}
\hline & ORR $(\%)$ & Leukopenia (\%) & Nausea and vomiting (\%) & KPS (\%) \\
\hline GP & 11.5 & 99.8 & 93.1 & 0.7 \\
\hline GP+Aidi & 79.0 & 52.2 & 48.3 & 37.7 \\
\hline GP+API & 42.5 & 20.0 & 31.3 & 74.6 \\
\hline GP+BJOE & 62.6 & 75.3 & 85.4 & 45.8 \\
\hline GP+CKSI & 68.6 & 46.3 & 56.9 & 59.8 \\
\hline GP+ELMI & 44.2 & 24.0 & 39.0 & - \\
\hline GP+KAI & 76.4 & 4.4 & 35.8 & 60.0 \\
\hline GP+KLT & 60.8 & 61.9 & 24.2 & 45.6 \\
\hline GP+LTNI & 27.1 & 36.0 & 45.5 & 64.7 \\
\hline GP+SQFZ & 48.5 & 63.2 & 49.5 & 71.0 \\
\hline GP+XAPI & 28.8 & 67.0 & 41.0 & 40.0 \\
\hline
\end{tabular}

Moreover, we paid more attention to anticancer drugs (ZC01) included Aidi, CKSI, XAIP, BJOE from the catalog of drugs for national basic medical insurance of China [18]. Aidi and CKSI showed better advantages in ranking of efficient and safety in this study. In contrast, GP regimen chemotherapy alone was comprehensively ranked as having the worst efficacy and safety of the treatment options (Figure 6).

\section{Subgroup and sensitivity analyses}

The included studies were divided into a long-term chemotherapy subgroup ( $>2$ cycles) and a short-term chemotherapy subgroup ( $\leq 2$ cycles). The results showed that the ORR of GP+BJOE decreased with a short-term cycle (Supplementary Table 3b), and the leukopenia advantage of GP+KAI (compared to other CHIs) was decreased (Supplementary Table $4 c)$. For evaluation criteria, we found that it did not exhibit a significant influence on the endpoint of the main outcomes (Supplementary Table 3a, Table 4b and Table $5 b$ ). In clinical treatment, GP regimen chemotherapy is mainly used for lung squamous cell carcinoma. A subgroup analysis was conducted after dividing the included studies into a mainly squamous cell carcinoma subgroup and a mainly adenocarcinoma subgroup. The results were consistent, except for the decreased efficacy of GP+CKSI (Supplementary Table 3c).

A previous study [19] reported that some CHIs may not exhibit a clear effect on adverse reactions without supportive treatment. Therefore, we divided the included studies involving adverse reactions into a supportive treatment subgroup and a no supportive treatment subgroup. In the no supportive treatment subgroup, the results showed that the leukopenia advantage of GP+KAI (compared to the other CHIs) was decreased (Supplementary Table 4a). Also, GP+CKSI, and GP+KAI showed no statistical differences in nausea and vomiting compared with GP regimen chemotherapy alone (Supplementary Table 5a).

To test the sensitivity of this NMA, we respectively excluded high-risk studies and not recommended dose studies. In the end, The SUCRAs ranking of different treatments outcomes were almost same as in Table 4. Therefore, the results of this meta-analysis are robust (Table 5).

Table 5. The sensitivity of this NMA

\begin{tabular}{|c|c|c|c|}
\hline & ORR (\%) & Leukopenia (\%) & Nausea and vomiting (\%) \\
\hline \multicolumn{4}{|c|}{ A. Exclusion of high-risk studies } \\
\hline GP & 12.2 & 99.7 & 92.1 \\
\hline GP+Aidi & 87.1 & 58.9 & 43.3 \\
\hline GP+API & 41.7 & 19 & 32.7 \\
\hline $\mathrm{GP}+\mathrm{BJOE}$ & 63.2 & 74.9 & 85.3 \\
\hline GP+CKSI & 69 & 44 & 53.5 \\
\hline GP+ELMI & 43.1 & 20.8 & 38.7 \\
\hline GP+KAI & 69.1 & 11.4 & 54.5 \\
\hline GP+KLT & 63.1 & 61 & 23.5 \\
\hline GP+LTNI & 24.9 & 33.9 & 43.6 \\
\hline GP+SQFZ & 48.1 & 60.8 & 40.9 \\
\hline GP+XAPI & 28.5 & 65.5 & 41.9 \\
\hline \multicolumn{4}{|c|}{ B. Exclusion of not recommended doses studies } \\
\hline GP & 12.5 & 99.7 & 91.3 \\
\hline GP+Aidi & 81.3 & 52.5 & 49.1 \\
\hline GP+API & 42.3 & 21.6 & 32.3 \\
\hline GP+BJOE & 66.2 & 65.3 & 89.4 \\
\hline GP+CKSI & 68.5 & 46.1 & 57.8 \\
\hline GP+ELMI & 42.8 & 24.4 & 39.8 \\
\hline GP+KAI & 76.9 & 4.3 & 35.4 \\
\hline GP+KLT & 56.1 & 67.2 & 26.4 \\
\hline GP+LTNI & 23.9 & 36.4 & 44.4 \\
\hline GP+SQFZ & 49.9 & 64.5 & 41 \\
\hline GP+XAPI & 29.6 & 68.2 & 43.2 \\
\hline
\end{tabular}

\section{Inconsistency and publication bias}

The publication bias was detected by qualitatively based on funnel-plot asymmetry (Figure 7) and quantitatively based on Egger's test (Figure 8). 
The points with different colors represent different comparisons between the interventions. We found possible publication bias, included nausea and vomiting $(\mathrm{P}=0.007)$, leukopenia $(\mathrm{P}=0.000)$. The Trim
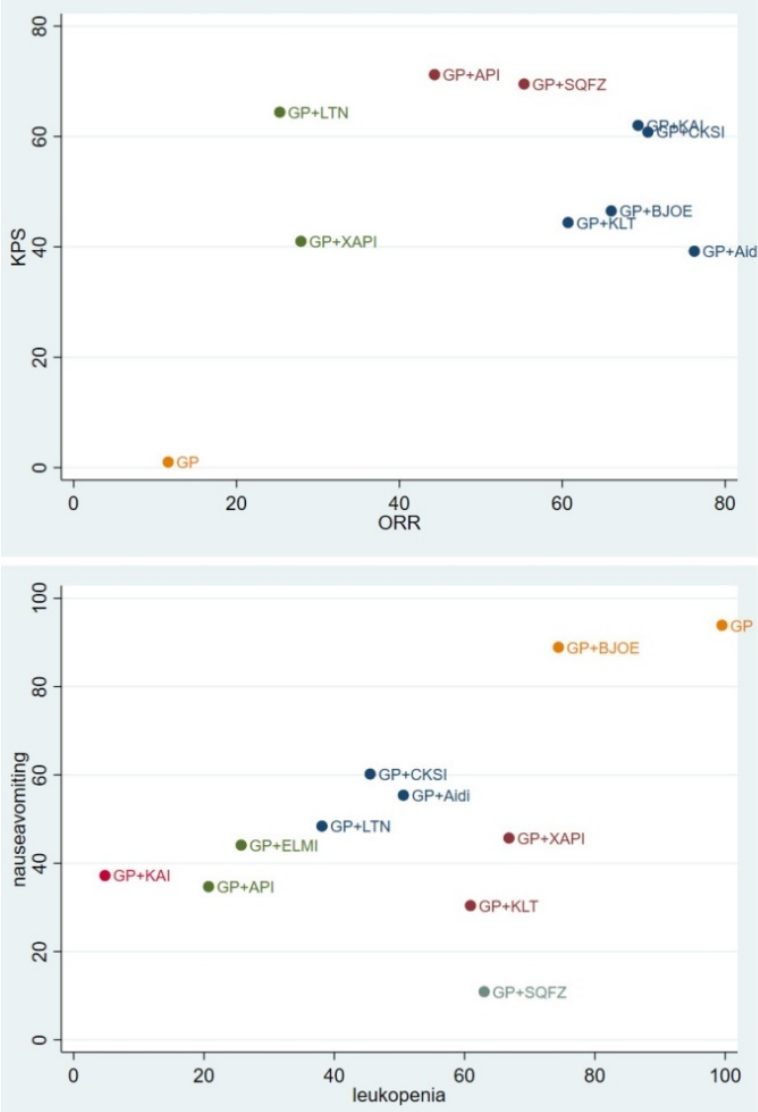

and Fill Method was used to further sensitivity analyses, the result showed there was no indications of publication with the Duval's trim and fill method (no new studies added).
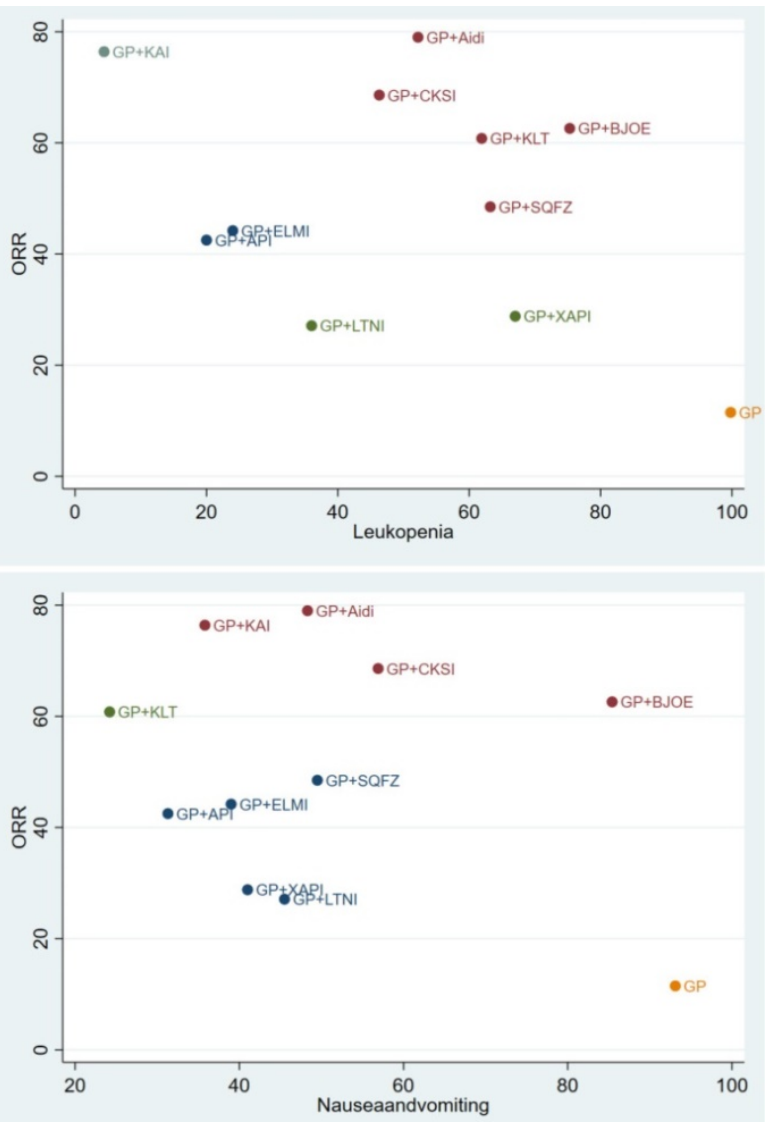

Figure 6. Cluster analysis plots. NOTE: Interventions in the upper-right section of the cluster analysis plots were more easy Leading to relevant results. GP: cisplatin and gemcitabine; Aidi: Aidi injection; KLT: Kanglaite injection; CKSI: Compound Kushen injection; BJOE: Brucea javanica Oil Emulsion injection; SQFZ: Shenqi Fuzheng injection; KAI: Kangai injection; XAPI: Xiaoaiping injection; API: Astragalus polysaccharide injection.
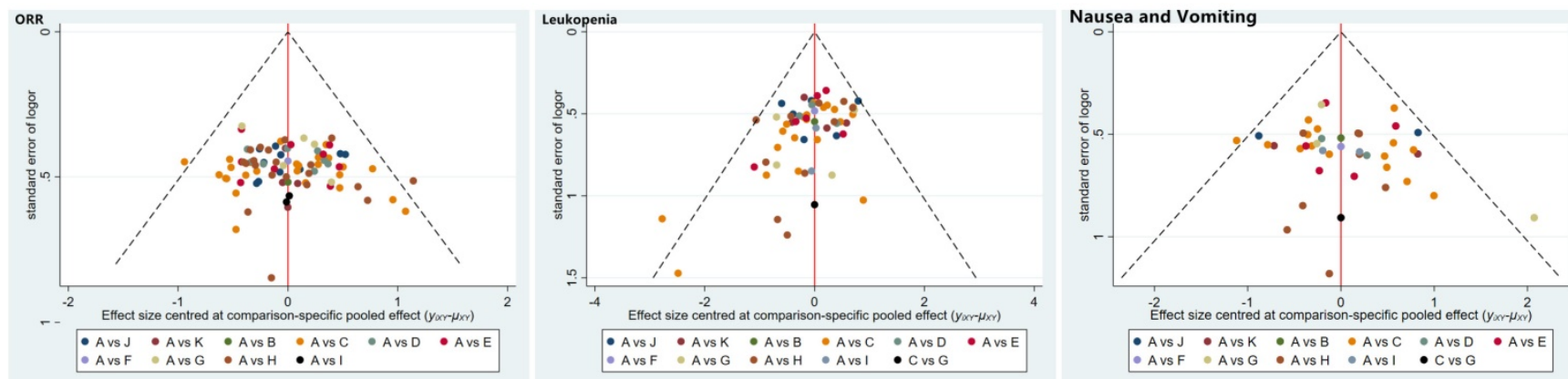

Figure 7. Funnel plots of the included randomized controlled trials.
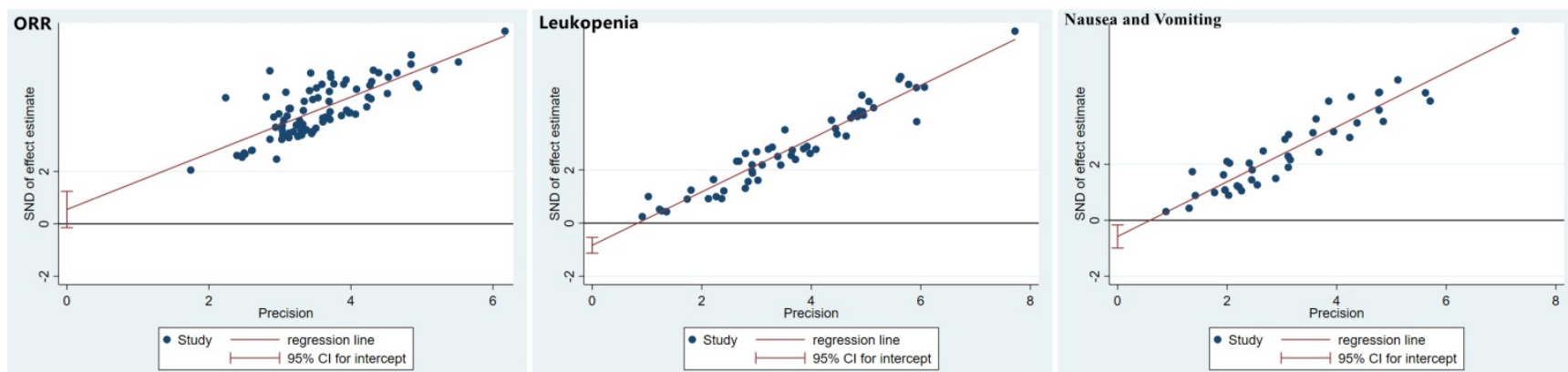

Figure 8. Egger's funnel plot with pseudo- $95 \%$ confidence limits. 


\section{Discussion}

NSCLC is a common respiratory malignancy worldwide. Although platinum-based chemotherapy has improved the clinical efficiency of NSCLC treatment, complementary therapy is still sought to reduce adverse reactions and improve the QOL of patients. Currently, TCM has been applied in multifaceted approaches and plays an indispensable role in the prevention and treatment of cancer, owing to its unique treatment concepts, theory, methods, and basic and clinical research [20]. In clinical therapy, CHIs have become known for their rapid efficacy and convenient application characteristics compared to TCM decoctions. However, due to the large number of CHIs and the lack of direct control studies, it is difficult to select an optimal scheme of CHIs combined with GP regimen chemotherapy for the treatment of advanced NSCLC. Hence, we conducted a NMA to evaluate clinical efficiency, adverse reactions, QOL, and long-term synergistic efficacy in stage III/IV NSCLC patients treated with GP chemotherapy plus CHIs.

This study included 92 RCTs with 10 CHIs used in the treatment of NSCLC. Aidi, KLT, CKSI and KAI showed obvious advantages in both efficacy and safety, and among them, Aidi+GP $(79.0 \%)$ showed great advantages of ORR, and KAI+GP and KLT+GP had the lowest probability in terms of leukopenia $(4.4 \%)$ and nausea and vomiting (24.2\%). KAI+GP might be the best performing combination. Based on the SUCRA analyses, this combination ranked first for reducing leukopenia, and was in the top three for improving ORR, reducing nausea and vomiting. Previous studies have demonstrated that KAI improves the effect and enhances QOL when used as an adjuvant treatment with chemotherapy. Such findings have been observed among patients with primary breast cancer [21], colorectal cancer [22], and NSCLC [23]. Recent pharmacological studies have reported that flavonoids from Astragalus membranaceus (Fisch.) Bge. var. mongholicus (Bge.) Hsiao and rare ginsenosides are the major effective substances of KAI [24]. It has also been reported that the flavonoid of Astragalus could inhibit the proliferation of $\mathrm{K} 562$ cells, and inhibit the tumor growth of C57 black (BL)/ 6 tumor-bearing mice by regulating immune $[25,26]$. Furthermore, ginseng can inhibit tumor cell growth and differentiation, increase the sensitivity of chemotherapy drugs, and enhance the immune function of peripheral blood lymphocytes in patients with cancer [27]. Besides, we also noticed anticancer drugs and adjuvant drugs for cancer got different advantages in the curative effects, such as high ORR of Aidi+GP. Through the cluster analysis, Aidi and CKSI showed better advantages than other anticancer drugs in ranking of efficient and safety in this study.

Differences in survival rates are of utmost importance to both clinicians and NSCLC patients. We are similarly interested in the long-term synergistic efficacy of CHIs compared with GP regimen chemotherapy alone. There were five $\mathrm{CHIs}$ (Aidi, KLT, CKSI, XAPI and ELMI) included in this NMA for which MST was reported, and KLT+GP was the only combination that showed a positive effect on MST compared with GP regimen chemotherapy alone.

GP regimen chemotherapy exhibits varying degrees of blood and gastrointestinal toxicity, and we selected the most common adverse clinical reactions, including nausea, vomiting, and leukopenia, to evaluate the role of CHIs in the prevention of adverse reactions. As the only $\mathrm{CHI}$ recommended for the treatment of leukopenia following chemotherapy [28], KAI also showed a clear advantage over other CHIs in this study. However, subgroup analyses found that, in the no supportive treatment subgroup, the leukopenia advantage of GP+KAI over other CHIs was decreased, and that GP+Aidi, GP+CKSI, and GP+KAI did not show statistical differences in nausea and vomiting compared with GP alone. The results of the subgroup analyses suggested that CHIs might have a limited effect in reducing adverse reactions. The results of the pathological pattern subgroup showed that CHIs might have a similar effect in squamous cell carcinoma and adenocarcinoma.

This study is the first to evaluate the efficacy and safety of different CHIs combined with GP regimen chemotherapy in the treatment of advanced NSCLC. Also, the rankings of CHIs according to their clinical effectiveness rates and other outcomes provides direction for clinical medication. However, there are some limitations of this study that should be noted. Firstly, each API+GP and ELMI+GP included only one study $[106,109]$ on this study, which may limit the strength of the evidence. Secondly, some included trials were of low methodological quality. There were 43 studies reported the random allocation methods, but no study provided detailed information on the random allocation concealment. Additionally, all of the included RCTs were performed in China, which reduces the universal applicability of the results. Finally, a potential limitation was publication bias, due to the fact that studies obtaining optimistic results could be more easily published than studies with unfavorable results. Though the existence of publication bias, sensitivity analyses of Trim and Fill Method showed the result was reliable.

It should be noted that evidence concerning the long-term synergistic efficacy of different CHIs 
combined with GP regimen chemotherapy, such as overall survival and progression-free survival, is still insufficient. And most studies did not mention the stages of chemotherapy, which may affect the efficacy. Therefore, the studies with high methodological quality are still required. And we appeal to clinical researchers to clarify the details of chemotherapy, include short-term and long-term synergistic efficacy with the specific normative data type and regard it as a vital outcome in future research.

\section{Conclusions}

In this network meta-analysis, KAI+GP of adjuvant drugs, Aidi+GP and CKSI+GP of anticancer drugs appeared to be the advantageous treatment options for patients with advanced NSCLC, owing to its superior therapeutic performance and reduced adverse reactions. Also, KLT+GP positively affected MST. However, many shortcomings in clinical trial methodology resulted in an inadequate assessment of clinical efficacy and safety. Therefore, direct and diverse multicenter comparisons between different CHIs are warranted to further confirm these results, and present an in-depth review in the near future.

\section{Supplementary Material}

Supplementary figures and tables. https://www.jcancer.org/v13p0465s1.pdf

\section{Acknowledgements}

We would like to thank Session of Evidencebased Clinical Club (EBC) for the direction on the manuscripts.

\section{Funding}

This work was financially supported by National key research and development plan of China [No. 2018YFC1707405] and National Natural Science Foundation of China [No. 81774289, 81473463]. The funders had no role in study design, data collection and analysis, decision to publish, or preparation of the manuscript.

\section{Author Contributions}

Conceptualization: Juan Li, Guang-Hui Zhu; Data curation: Tong-Tong $\mathrm{Liu}$, Bo-Wen $\mathrm{Xu}$; investigation: Bo-Wen $\mathrm{Xu}$, Guang-Hui Zhu; Methodology: Juan Li, Guang-Hui Zhu, Tong-Tong Liu; Supervision: Jie Li; Writing - original draft: Juan Li, Guang-Hui Zhu; Writing - review \& editing: Juan Li.

\section{Ethical statement}

The authors are accountable for all aspects of the work in ensuring that questions related to the accuracy or integrity of any part of the work are appropriately investigated and resolved.

\section{Data availability statement}

All data relevant to the study are included in the article or uploaded as supplementary information.

\section{Competing Interests}

The authors have declared that no competing interest exists.

\section{References}

1. LI Y, GUO QS. Recearch progress of maintenance therapy in advanced non-

small cell lung cancer. Chinese Journal of Cancer Prevention and Treatment. 2014; 21(10): 800-804.

2. FOSS KM, SIMA C, UGOLINI D, et al. miR-1254 and miR-574-5p Serum-Based microRNA Biomarkers for Early-Stage Non-small Cell Lung Cancer. Journal of thoracic oncology: official publication of the International Association for the Study of Lung Cancer. 2011; 6(3): 482-488.

3. LE CT. Adjuvant chemotherapy for resectable non-small cell lung cancer: where is it going? Ann Oncol. 2010; 21(7): 196-198.

4. Scagliotti GV, Parikh P, von Pawel J, et al. Phase III study comparing cisplatin plus gemcitabine with cisplatin plus pemetrexed in chemotherapy-naive patients with advanced-stage non-small-cell lung cancer. J Clin Oncol. 2008; 26(21): 3543-3551.

5. Ender Dogan, Mahmut Ucar, Teoman Sakalar, et al. Biweekly cisplatin and gemcitabine with two different doses in non-small cell lung cancer patients: A retrospective singlecenter experience. J BUON. 2018; 23(4): 1070-1076.

6. Dasari S, Tchounwou PB. Cisplatin in cancer therapy: molecular mechanisms of action. Eur J Pharmacol. 2014; 740: 364-378.

7. Xiao Z, Wang C, Zhou M, et al. Clinical efficacy and safety of Aidi injection plus paclitaxel-based chemotherapy for advanced non-small cell lung cancer: A meta-analysis of 31 randomized controlled trials following the PRISMA guidelines. J Ethnopharmacol. 2019; 228: 110-122.

8. Chen H, Yao X, Liu Z, et al. Efficacy and safety of Shenqi Fuzheng injection combined with platinum-based chemotherapy for stage III/IV non-small cell lung cancer: A protocol for systematic review and meta-analysis of randomized controlled trials. Medicine (Baltimore). 2019; 98(39): e17350.

9. Xiaoju Bai. Analysis of anti-tumor varieties of traditional Chinese medicine injections. Pharmacology and Clinics of Chinese Materia Medica. 2009; 25(03): 72-73.

10. Xiao-Wei $\mathrm{Xu}$, Zheng-Zhong Yuan, Wen-Hao Hu, et al. Meta-analysis on elemene injection combined with cisplatin chemotherapeutics in treatment of non-small cell lung cancer. Zhong Guo Zhong Yao. 2013; 38(9): 1430-7.

11. Chenchen Zhao, Haifeng Yan, Wentai Pang, et al. Lentinan combined with cisplatin for the treatment of non-small cell lung cancer. Medicine (Baltimore). 2021; 100(12): e25220.

12. Hutton B, Salanti G, Caldwell DM, et al. The PRISMA extension statement for reporting of systematic reviews incorporating network meta-analyses of health care interventions: checklist and explanations. Ann Intern Med. 2015; 162: 777-784.

13. Cuccurullo V. AJCC cancer staging handbook: from the AJCC cancer staging manual (7th edition). J Eur J Nuclear Med. 2011; 38: 408-1408.

14. [Internet] G.S. Higgins JPT, Cochrane Handbook for Systematic Reviews of Interventions Ver-sion 5. 1. 0. http://training.cochrane.org/ handbook.

15. A. B. Miller, B. Hoogstraten, M. Staquet, et al. Reporting results of cancer treatment. Cancer. 1981; 47(1): 207-214.

16. H. Watanabe, S. Yamamoto, H. Kunitoh, et al. Tumor response to chemotherapy: Te validity and reproducibility of RECIST guidelines in NSCLC patients. Cancer Science. 2003; 94(11): 1015-1020.

17. Xiaoyu Zheng. Guiding principles for clinical research of new Chinese medicine (trial). Beijing: China medical science and technology press. 2002: 216-221.

18. Chen B, Wang Z, Zhou LP, et al. Clinical Effects of Aidi Injection Combined with Chemotherapy of GP Regimen in Treating Patients with Advanced Non-Small Cell Lung Cancer. J Chinese Archives of Traditional Chinese Medicine. 2014; 32(07): 1666-1668. 
19. Fu LJ. Analysis of the clinical efficacy of Aidi injection combined with GP chemotherapy on elderly advanced non-small cell lung cancer. Guide of China Medicine. 2012; 10(26): 466.

20. Gen KJ, Dong JG, Su H, et al. Observation on the efficacy and safety of Aidi injection combined with gemcitabine and cisplatin regimen in the treatment of advanced non-small cell lung cancer. Henan Medical Research. 2020; 29(12): 2231-2232.

21. Guo X. Effect analysis of Aidi injection combined with GP regimen in the treatment of advanced NSCLC. Practical Clinical Journal of Integrated Traditional Chinese and Western Medicine. 2020; 20(04): 104-105.

22. Huang WJ, Zheng JQ, Guo BL. The clinical efficacy of Aidi injection combined with GP regimen in the treatment of advanced non-small cell lung cancer and its effect on the immune function of patients. The Journal of Medical Theory and Practice. 2017; 30(07): 995-997.

23. Kuang XK, Zhu XL, Wang LP, et al. Aidi Plus Gemcitabine and Cisplatin for Patients with Advanced Non-Small Cell Lung Cancer. Journal of Basic and Clinical Oncology. 2008; 21(04): 305-307.

24. Li J, Yang ZW, Song X, et al. Study on the short-term efficacy and safety of Aidi injection assisted GP regimen in intervention of advanced non-small cell lung cancer. Hebei Medical Journal. 2016; 38(09): 1345-1347.

25. Liu, Y., Liu, Y., and Zhang, T. Aidi Injection and Chemotherapy for Advanced Non-small Cell Lung Cancer: a Short-Term Curative Effect Study. Natl. Med Front China. 2010; 5(19): 46

26. Liu HF, Nie QH, Lu YJ, et al. The clinical effect of Aidi injection combined with GP regimen on advanced non-small cell lung cancer and its effect on serum VEGF expression and immune function. Jiangxi Journal of Traditional Chinese Medicine. 2019; 50(03): 42-44.

27. Li XY. The Comparative study in Aidi injection and Kang ai injection in combination with chemotherapy for middle-aged and elderly patients who suffered from advanced non-small cell lung cancer. M.Sc. Thesis, Fujian University of Traditional Chinese Medicine. 2015.

28. Liu YH, Zhao YY. Efficacy analysis of Aidi injection combined with GP regimen on advanced non-small cell lung cancer. Chinese Journal of Practical Medicine. 2014; 41(24): 67-69.

29. Ma M. The effect of Aidi injection combined with GP chemotherapy in the treatment of advanced NSCLC patients with deficiency of both qi and yin and its effect on the immune function and coagulation function of the patient. Asia-Pacific Traditional Medicine. 2017; 13(21): 108-110.

30. Shen RR, Zhang YY, Yu P, et al. Effect of Aidi injection combined with gemcitabine and cisplatin in patients with advanced lung cancer. Modern Medicine and Health Research Electronic Journal. 2021; 5(08): 29-31.

31. Song ZZ, Liu XL, Zhang Yh. Aidi injection combined with GP regimen in the treatment of advanced non-small cell lung cancer. Medical Research and Education. 2009; 26(05): 36-37.

32. Su SJ. Aidi injection combined with gemcitabine and cisplatin in the treatment of advanced non-small cell lung cancer. Chinese Journal of Practical Medicine. 2017; 44(24): 112-115.

33. Sun GS, Liu JR, Wang TT, et al. Aidi injection combined with Zephyr plus cisplatin regimen in the treatment of 33 cases of advanced non-small cell lung cancer. Fujian Medical Journal. 2008; 30(06): 134.

34. Sun JB, Xu PR, Shi DL, et al. Evaluation on clinical efficacy of Aidi injection combined with GP chemotherapy in treatment of advanced non-small cell lung cancer in elder patients. Journal of Jilin University (Medicine Edition). 2012; 38(01): 151-154.

35. Wang SD. Effect of Aidi injection on serum levels of interleukin and tumor necrosis factor in patients with lung cancer chemotherapy. Modern Diagnosis and Treatment. 2015; 26(10): 2256-2257.

36. Wen HQ. Observation on Therapeutic Effect of Integrated Traditional Chinese and Western Medicine in Treating Elderly Advanced Non-small Cell Lung Cancer. Modern Journal of Integrated Traditional Chinese and Western Medicine. 2014; 23(21): 2325-2326.

37. Wen K, Li J, Peng DY. Observation on the curative effect of Aidi injection combined with GP regimen in the treatment of advanced non-small cell lung cancer. Chinese Journal of Basic Medicine in Traditional Chinese Medicine. 2009; 15(09): 716-717.

38. Wu, T, Chen, S. Research on Feasibility of Aidi Injection and Chemotherapy in the Non-small Cell Lung Cancer. China Foreign Med. Treat. 2017; 15(36): 126-127.

39. Xu H, Yang T, Zhou M. Clinical evaluation of Aidi injection combined with GP regimen in the treatment of advanced non-small cell lung cancer. International Journal of Respiration. 2013; 33(10): 765-767.

40. Xu Y, Zhang YJ, Lu BZ. Clinical Study on Aidi Injection Combined Chemotherapy for Treating Middle and Late Stage Lung Cancer. China Pharmaceuticals. 2012; 21(23): 56-57.

41. Xu ZJ. Efficacy of Aidi injection adjuvant GP chemotherapy regimen in patients with advanced non-small cell lung cancer. Henan Medical Research. 2020; 29(27): 5133-5135.
42. Zhang L. Clinical observation of Aidi combined with GP regimen in the treatment of advanced non-small cell lung cancer. The Journal of Practical Medicine. 2009; 25(17): 2929-2930.

43. Zhang XC. Clinical Observation of Addie Injection Combined with GP Regimen in the Treatment of Non-Small Cell lung cancer. Journal of Liaoning University of Traditional Chinese Medicine. 2016; 18(02): 173-175.

44. Zhao J, Li J. Application effect of Aidi injection combined with GP scheme in advanced non-small cell lung cancer. Clinical Research and Practice. 2019; 4(25): 117-119.

45. Zhao S, Zhang Q, Wang DX. Efficacy analysis of Aidi injection in combination with chemotherapy on immunity in patients with lung cancer. Medical Journal of West China. 2015; 27(11): 1663-1666.

46. Zhou DM. Analysis of the clinical efficacy of Aidi injection combined with gemcitabine and cisplatin (GP) regimen in the treatment of advanced non-small cell lung cancer. Journal of Clinical Medical Literature. 2018; 5(1): 47-48.

47. Bao $\mathrm{H}, \mathrm{Ma} \mathrm{N}$, Qin NN. Clinical efficacy and safety of Kanglaite combined with gemcitabine and cisplatinum chemotherapy in the treatment of advanced non-small cell lung cancer. J Special Health. 2019; (20): 66-7.

48. Chen C. Non-small Cell Lung Cancer Randomized Parallel Controlled Study of Advanced Kanglaite Injection combined with GP in Treatment. Journal of Practical Traiditional Chinese Internal Medicine. 2018; 32(03): 46-48.

49. Chen W, Wei T. Clinical Effect and the Influence on Immune Function of Kanglaite Injection combined with Gemcitabine and Cisplatin in the Treatment of Advanced Non-small Cell Lung Cancer. Chinese Journal of Clinical Oncology and Rehabilitation. 2016; 23(07): 814-817.

50. Chen Y. Effect of kanglaite combined with Chemotherapy of GP in Patients with advanced non-small cell lung cancer. Medical Journal of Chinese People's Health. 2018; 30(08): 73-75.

51. Guan XQ, Liu JW, Li L. Clinical Observation of Coix Seed Oil for Injection combined with GP Regimen in the Treatment of Advanced Non-small Cell Lung Cancer. Chinese Journal of Postgraduates of Medicine. 2009; 32(16): 60-62

52. Gui XM, Dai L,Yuan QW, et al. Effects of Kanglaite Injection on Levels of $\mathrm{CD}+3, \mathrm{CD}+4, \mathrm{NK}$ and $\mathrm{CD}+4 / \mathrm{CD}+8$ in Patients with Advanced Non-small Cell Lung Cancer. Chinese Archives of Traditional Chinese Medicine. 2020; 38(05): 147-150.

53. Huang Zhenbu, Zhu Chunya, Wu Shixiu. Effect of Kanglaite Combined with Chemical Therapy Advanced Non-small Cell Lung Cancer. China Pharmacy. 2010; 21(20): 1860-1861.

54. Li Hongyun. Clinical Effect of Kanglaite Injection Combined with Gemcitabine and Cisplatin on Advanced Non-small-cell Lung Cancer and the Impact on Immune Function. Practical Journal of Cardiac Cerebral Pneumal and Vascular Disease. 2017; 25(05): 121-123.

55. Liang SG, Dong YH, Wang J, et al. Kanglaite Combined with GP in the Treatment of 25 cases of Senile Non-small-cell Lung Carcinoma. China Journal of Chinese Medicine. 2014; 29(01): 11-12.

56. Liu Fang. Clinical Observation on the Treatment of Advanced Non-small Cell Lung Cancer by Kanglaite combined with GP. Research of Integrated Traditional Chinese and Western Medicine. 2019; 11(01): 36-38.

57. Liu Junqiang, Shang Liqun, Li Xuechang, et al. Effect of kanglaite combined with Chemical Therapy Advanced Non-small Cell Lung Cancer. Journal of Modern Oncology. 2011; 19(10): 1974-1976.

58. Liu Yang, Ma Ping. Therapeutic Effect of Kanglaite Injection combined with Gemcitabine and Cisplatin on Advanced Non-small Cell Lung Cancer. Chinese Journal of Practical Medicine. 2015; 42(4): 43-45.

59. Long Shenggen, Xiao Yulan. Clinical Efficacy of Kanglaite combined with GP Regimen in Patients with Advanced NSCLC and Its Effect on Various Indexes of Immune Function. Anti-Infection Pharmacy. 2017; 14(02): 377-380.

60. Sun Siqing, Zhu Xiaoli, Huang Jing, et al. Effect of Kanglaite Injection combined with Chemotherapy for Improvement of Quality of Life in Advanced Stage NSCLC Patients. Journal of Practical Oncology. 2012; 27(05): 506-510

61. Wang Lei. Effects of Kanglaite Adjuvant Chemotherapy on Immune Function of Non-small Cell Lung Cancer and Clinical Efficacy Analysis. Laboratory Medicine and Clinic. 2014; 11(08): 1072-1073+1075.

62. Wang Yuan, Hui Shuang, Li Min, et al. Clinical trial of Kanglaite Injection in Gemcitabine combined Cisplatin Regimen Chemotherapy for Advanced Non-small Cell Lung Cancer. The Chinese Journal of Clinical Pharmacology. 2017; 33(23): 2354-2356+2360.

63. Yan Qiaohui, Xing Guochen, Pan Qiong. Effect Observation of GP Regimen combined with Kanglaite in the Treatment of Advanced Non-small Cell Lung Cancer. Practical Clinical Journal of Integrated Traiditional Chinese and Western Medicine. 2018; 18(09): 112-113+121. 
64. Yao Jun, Song Xiao. Efficacy and Safety Analysis of GP Scheme combined with Kanglaite Injection for Advanced Non-small Cell Lung Cancer. Journal of Clinical and Experimental Medicine. 2017; 16(12): 1195-1198.

65. Ye Chengyuan, Zhao Fangchao, Xing Shushan, et al. Clinical Observation of Kanglaite Injection Combined with Gemcitabine Cisplatin Regimen for Advanced Non-small Cell Lung Cancer. Clinical Research. 2019; 27(07): 24-26.

66. Zhang Miaomiao. Clinical Efficacy and Safety of Kanglaite Injection in Patients with Advanced Non-small Cell Lung Cancer. The Journal of Medical Theory and Practice. 2019; 32(01): 54-56.

67. Duan P, Xiao MW, Xiao W, et al. Efficacy of compound Kusheng injection in combination with chemotherapy in patients with advanced non-small cell lung cancer. Chinese Journal of New Drugs. 2009; 18(18): 1760-1763.

68. Fan QL. Effect of the non-small cell lung cancer patients treated with Sophora Injection and analyze its impact on the quality of life of patients. China Medicine and Pharmacy. 2015; 5(21): 95-97.

69. Feng Q, Zheng HY. Clinical Evaluation of Compound Kushen Injection Combined with GP in the Treatment of Patients with Different Stages of Non-small Cell Lung Cancer. World Chinese Medicine. 2018; 13(05): 1164-1167+1171.

70. Gao LJ. Clinical Study of Yanshu Compound Kushen Injection in Treatment of Lung Cancer. Liaoning Journal of Traditional Chinese Medicine. 2019; 46(12): 2583-2585.

71. Liu Y, Wei H, Meng LZ, et al. Clinical Observation of Yan-shu Injection and GP Chemotherapy on Non-Small Cell Lung Cancer in Advanced Stage. Shanxi Journal of Traditional Chinese Medicine. 2009; 25(08): 24-25.

72. Lu WL. Efficacy of Compound Matrine Injection in Combination with Gemcitabine and Cisplatin Chemotherapy in the Treatment of Patients with Advanced Non-small-cell lung Cancer. Liaoning Journal of Traditional Chinese Medicine. 2017; 44(06): 1214-1215.

73. Wang ZX, Li J, Lu BB, et al. Efficacy and Detoxicating Effect of Compound Matrine Injection in Combination with Gemcitabine and Cisplatin Chemotherapy in the Treatment of Patients with Advanced Non-small-cell Lung Cancer. Evaluation and Analysis of Drug-Use in Hospitals of China. 2009; 9(12): 932-933.

74. Zhang MY, He W, Zhang HF, et al. Efficacy of adjuvant treatment with sophora flavescens injection for advanced non-small cell lung cancer. Chinese Journal of Clinical Oncology and Rehabilitation. 2019; 26(03): 327-329.

75. Zhou HY. Fufang Kushen Zhusheye with Gemcitabine and Cisplatin for Curing of Advanced Non-Small Cell Lung Cancer. Chinese Journal of Clinical Medicine. 2011; 18(04): 489-491.

76. Chen HL, Wang WP, Lan YP, et al. Effect of Fructus Brucea Oil Emulsion Combined with Chemotherapy of GP in Patients with Advanced NSCLC. Journal of Practical Oncology. 2010; 25(5): 584-586.

77. Liu SR, Li XH, Liu J, et al. Effects of brucea javanica oil emulsion injection on the cisplatin chemotherapy regimen in the treatment of non-small cell lung cancer and its toxic and side. Clinical Research and Practice. 2019; 4(29): 56-58.

78. Su BK. Clinical Observation on the Effect of Brucea Javanica Oil-emulsion Injection on the Chemotherapy of GP in Patients with Stasis Lung Collateral Syndrome in Advanced NSCLC. M.Sc. Thesis, Fujian University of Traditional Chinese Medicine. 2017.

79. Tian L, Wang CY. Brucea Javanica Oil Emulsion Injection Combined with GP Regimen in Treatment of Advanced Non-small Cell Lung Cancer. Acta Chinese Medicine. 2017; 32(03): 339-341.

80. Wang JH, Li GP, Gong S. Clinical study of GP regimen combined with Brucea javanica oil emulsion in the first-line treatment of advanced non-small cell lung cancer. Chinese Journal of Postgraduates of Medicine. 2012; 35(24): 11-13.

81. Wang LC, Men XL, Gao SC. Clinical Observation on the Effect of Brucea Javanica Oil Emulsion Combined with Chemotherapy in the Treatment for Middle-advanced Nonsmall-cell Lung Cancer. Medical Recapitulate. 2015; 21(05): 879-881.

82. Wang YZ. Effect of Brucea javaneae oil emulsion on quality of life in elderly patients with non-small cell lung cancer. Special Health. 2021; (16): 22-23

83. Ye HN, Qin BL, Su M, et al. Clinical effect of Brucea javanica oil emulsion combined with chemotherapy in the treatment of advanced non-small cell lung cancer. Guiding Journal of Traditional Chinese Medicine and Pharmacy. 2015;21(07):35-38.

84. Yu HW, Zhang YB, Zhang L, et al. Effect of Brucea javaneae oil emulsion combined with GP in the treatment of lymph node metastasis of non-small cell lung cancer. China Practical Medicine. 2020; 15(34): 108-110.
85. Zhang B, Chen MX, Chen HY, et al. Effect of Fructus Bruceae oil emulsion on peripheral blood $\mathrm{T}$ lymphocyte subsets and the quality of life in elderly patients with NSCLC. Shanxi Medical Journal. 2017; 46(12): 1402-1404.

86. An AJ, Cao JJ. Intervention Effect of Shenqi Fuzheng Injection on Cancerrelated Fatigue in the Patients with Non-Small Cell Lung Cancer during Chemotherapy. Medical Innovation of China. 2014; 11(28): 25-27.

87. He WJ, Zhao JQ. Clinical Observation of Shenqi Fuzheng Injection Combined with GP Regimen to Treat Patients with Advanced Non-small Cell Lung Cancer. Medical Recapitulat. 2008; (12): 1756+1921.

88. He WX. Effect of Shenqi Fuzheng injection assisted GP regimen on advanced non-small cell lung cancer. Practical Clinical Journal of Integrated Traditional Chinese and Western Medicine. 2021; 21(11): 44- $45+131$.

89. Lin CH. Clinical analysis of Shenqi Fuzheng injection combined with GP regimen in the treatment of stage III/IV non-small cell lung cancer. M.Sc. Thesis, China Academy of Chinese Medical Science. 2014.

90. Lou T, Shao SW. Clinical observation of Shenqi Fuzheng injection combined with chemotherapy in elderly patients with advanced non-small cell lung cancer. Modern Practical Medicine. 2020; 32(06): 632-634.

91. Luo BP, Zhou Y, Cao T, et al. Clinical trial of Shenqi Fuzheng injection on quality of life in patients with non-small cell lung cancer after chemotherapy. The Chinese Journal of Clinical Pharmacology. 2018; 34(18): 2137-2139.

92. Wang YQ, Jiang XF. Clinical Observation of Shenqi Fuzheng Injection Combined with Chemotherapy in the Treatment of Advanced Lung Cancer. Practical Clinical Medicine. 2010; 11(04): 25-26+35.

93. Yao DJ, Cai Y, Chen Y. Clinical observation of Shenqi Fuzheng injection combined with GP regimen in the treatment of advanced non-small cell lung cancer. Chinese Journal of Clinical Research. 2013; 26(12): 1378-1379.

94. Zhang LM, Chen JH, Wang W, et al. Efficacy of Shenqifuzheng Injection in the Treatment of Advanced Non-small Cell Lung Cancer. World Clin. Med. 2017; 11(16): 113-114.

95. Zhou T, Liu SQ, Gao P, et al. Shenqifuzheng Injection Combined with GC Chemotherapy in the Treatment of 35 Patients of Advanced Non-small Cell Lung Cancer. Cancer Res. Clin. 2009; 21 (10): 706-707.

96. Dong H, Wang CD, Gong Q. Effects of Kangai injection adjuvant chemotherapy on immune function and tumor markers in elderly patients with advanced non-small cell lung cancer. Chinese Journal of Gerontology. 2019; 39(01): 52-55.

97. Yi CZ, Xu YH, Zhang D F, et al. Clinical Observation on the Effect of Kangai Injection on the Chemotherapy of GP in Elderly Patients with Advanced NSCLC. Guiding J. Tradit. Chin. Med. Pharm. 2011; 17(09): 24-26.

98. Jiang H, Zheng K, Tong LM. The effect of Kangai injection combined with gemcitabine + cisplatin chemotherapy on patients with advanced non-small cell lung cancer and the quality of life. Chinese Journal of Primary Medicine and Pharmacy. 2018; 25(11): 1447-1451.

99. Lu YZ, Tan BQ, Sun DP, et al. Clinical Observation of Kang'ai Injection Adjuvant Chemotherapy for Advanced Lung Cancer. China Pharmacy. 2017; 28(35): 4984-4987.

100. Shang LQ, Wang W, Li XC, et al. Application of Kangai injection in postoperative adjuvant chemotherapy for stage IIIA non-small cell lung cancer. Chinese Journal of Clinicians (Electronic Edition). 2011; 5(15): 4509-4511.

101. Tao HZ. Effect of gemcitabine and cisplatin combined with Kangai injection in the treatment of advanced non-small cell lung cancer and its effect on the quality of life of patients. Modern Practical Medicine. 2020; 18(13): 139-140.

102. Hu XL, Liu XS, Chen XD. Clinical evaluation of Xiaoaiping injection combined with gemcitabine and carboplatin in the treatment of stage IIIB and IV non-small cell lung cancer. Drug Evaluation Research. 2017; 14(02): 266-269.

103. Li QL, Chen B. Clinical Effects of Xiaoaiping Injection Combined with GP Chemotherapy Regimen in Treating Patients with Advanced Non-Small Cell Lung Cancer. Chinese Archives of Traditional Chinese Medicine. 2016; 34(04): 785-787.

104. Liu JR, An YH, Yang WB, et al. Clinical Study on Xiaoaiping Injection Combined with GP Regimen in the Treatment of Advanced Lung Squamous Cell Carcinoma. Medical Innovation of China. 2016; 13(13): 97-101.

105. Zhang FY, Li QW, Guan JZ, et al. Curative Observation of Xiaoaiping Injection Combined with GP Regimen in the Treatment of Patients with Advanced Non-Small Cell Lung Cancer. Journal of Basic and Clinical Oncology. 2011; 24(05): 415-417.

106. Qin ZQ, Lu LQ, Yuan GR, et al. Clinical Observation of Huangqiduotang Injection Combined with GP Regimen to Treat Patients with Advanced 
Non-small Cell Lung Cancer. Chinese Archives of Traditional Chinese Medicine. 2009; 27(03): 664-666.

107. Han L, Li JS, Liu Y. 25 cases of advanced non-small cell lung cancer treated with lentinan combined with GP regimen. Herald of Medicine. 2012; 31(08): 1029-1032.

108. Li JJ, Song WS, Wang SG. 30 patients with advanced non-small cell lung cancer treated with lentinan combined with gemcitabine and cisplatin chemotherapy. Guangdong Medical Journal. 2013; 34(09): 1432-1434.

109. Chu DJ, Guo SG, Yao DE. Elemene emulsion combined with GP regimen for advanced non-small cell lung cancer. China clinical Medicine. 2010; 17(04): 491-493.

110. Notice of the Ministry of human resources and social security of the state medical insurance administration on printing and distributing: Notice on the catalogue of drugs for national basic medical insurance, industrial injury insurance and maternity insurance (2020). Medical insurance [2020] No. 53. http://www.nhsa.gov.cn/art/2020/12/28/art 37_4220.html.

111. Li J, Li HZ, Zhu GH, et al. Efficacy and safety of Kanglaite injection combined with first-line platinum-based chemotherapy in patients with advanced NSCLC: a systematic review and meta-analysis of 32 RCTs. Ann Palliat Med. 2020; 9(4): 1518-1531.

112. Lin H, Liu J, Zhang Y. Developments in cancer prevention and treatment using traditional Chinese medicine Front Med. 2015; 5(2): 127-133.

113. Jing-Xian Xue, Zhi-Yuan Zhu, Wei-He Bian, et al. The Traditional Chinese Medicine Kangai Injection as an Adjuvant Method in Combination with Chemotherapy for the Treatment of Breast Cancer in Chinese Patients: A Meta-Analysis. Evid Based Complement Alternat Med. 2018; 18: 6305645

114. Siqi Huang, Weijun Peng, Dan Mao, et al. Kangai Injection, a Traditional Chinese Medicine, Improves Efficacy and Reduces Toxicity of Chemotherapy in Advanced Colorectal Cancer Patients: A Systematic Review and Meta-Analysis. Evid Based Complement Alternat Med. 2019; 8423037.

115. Hongxiao Li, Yuejin Ji, Shiping Zhang, et al. Kangai Injection Combined with Platinum-based Chemotherapy for the Treatment of Stage III/IV Non-Small Cell Lung Cancer: A Meta-analysis and Systematic Review of 35 Randomized Controlled Trials. Journal of Cancer. 2019; 10(21): 5283-5298.

116. Li P. Studies on the materials basis of medicinal effectiveness in Kang'ai injection. M.Sc. Thesis, Jilin University. 2019.

117. Dongqing Zhang, Yuan Zhuang, Jichun Pan, et al. Investigation of effects and mechanisms of total flavonoids of Astragalus and calycosin on human erythroleukemia cells. Oxid Med Cell Longev. 2012; 209843.

118. Bing Yang, Gui-Hong $\mathrm{Yu}$, Ming-Yu Li, et al. Mechanism of flavonoid components in Astragali Radix in inhibiting tumor growth and immunoregulation in C57BL/6 tumor bearing mice based on "invigorating Qi for consolidation of exterior". China Journal of Chinese Materia Medica. 2019; 44(23): 5184-5190.

119. Linming L, Yaning S, Yina J, et al. Advance in components with antitumor effect of Panax ginseng and their mechanisms. Chinese Traditional and Herbal Drugs. 2017; 48: 582-96.

120. Tian SD, Dong Q, Qi S, et al. Expert consensus on the prevention and treatment with Chinese medicine of leukopenia after chemotherapy. Modern Chinese Clinical Medicine. 2018; 3(25): 1-6. 